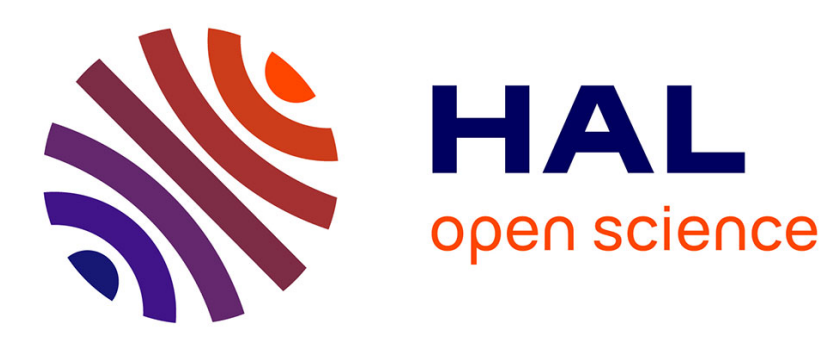

\title{
The stationary three-dimensional Navier-Stokes Equations with a non-zero constant velocity at infinity
}

Chérif Amrouche, Huy Hoang Nguyen

\section{To cite this version:}

Chérif Amrouche, Huy Hoang Nguyen. The stationary three-dimensional Navier-Stokes Equations with a non-zero constant velocity at infinity. 2008. hal-00206208

\section{HAL Id: hal-00206208 \\ https://hal.science/hal-00206208}

Preprint submitted on 16 Jan 2008

HAL is a multi-disciplinary open access archive for the deposit and dissemination of scientific research documents, whether they are published or not. The documents may come from teaching and research institutions in France or abroad, or from public or private research centers.
L'archive ouverte pluridisciplinaire HAL, est destinée au dépôt et à la diffusion de documents scientifiques de niveau recherche, publiés ou non, émanant des établissements d'enseignement et de recherche français ou étrangers, des laboratoires publics ou privés. 


\title{
The stationary three-dimensional Navier-Stokes Equations with a non-zero constant velocity at infinity
}

\author{
Chérif AMROUCHE ${ }^{\star}$ and Huy Hoang NGUYEN ${ }^{\dagger}$ \\ Laboratoire de Mathématiques Appliquées \\ CNRS UMR 5142 \\ Université de Pau et des Pays de l'Adour \\ IPRA - Avenue de l'Université 64013 Pau, France \\ * cherif.amrouche@univ-pau.fr \\ ${ }^{\dagger}$ huy-hoang.nguyen@etud.univ-pau.fr
}

\begin{abstract}
This paper is devoted to some mathematical questions related to the 3-dimensional stationary Navier-Stokes. Our approach is based on a combination of properties of Oseen problems in $\mathbb{R}^{3}$.
\end{abstract}

Keywords: Navier-Stokes equations; Oseen equations; weighted Sobolev spaces; fluid mechanics.

AMS class: 35Q30, 76D03, 76D05, 76D07

\section{Introduction}

Let $\Omega^{\prime}$ be a bounded open region of $\mathbb{R}^{3}$, not necessarily connected, with a Lipschitz-continuous boundary and let $\Omega$ be the complement of $\overline{\Omega^{\prime}}$. We suppose that $\Omega^{\prime}$ has a finite number of connected components and each connected component has a connected boundary, so that $\Omega$ is connected. The problem consists then in finding a velocity field $\boldsymbol{u}=\left(u_{1}, u_{2}, u_{3}\right)$ and the pressure $\pi$ satisfy the Navier-Stokes system:

$$
(\mathcal{N S}) \begin{cases}-\nu \Delta \boldsymbol{u}+\boldsymbol{u} . \nabla \boldsymbol{u}+\nabla \pi=\boldsymbol{f} & \text { in } \Omega, \\ \operatorname{div} \boldsymbol{u}=0 & \text { in } \Omega, \\ \boldsymbol{u}=0 & \text { on } \partial \Omega, \\ \boldsymbol{u} \rightarrow \boldsymbol{u}_{\infty} & \text { at infinity }\end{cases}
$$

where $\nu>0, f$ and $\boldsymbol{u}_{\infty} \in \mathbb{R}^{3}$ are respectively the viscosity of the fluid, the external force field acting on the fluid and a given constant vector. The third equation of the system states that the fluid adheres at the surface of the body, which is the common no-slip condition. For the last equation, we have two different cases concerning the behavior of $\boldsymbol{u}$ at infinity. If $\boldsymbol{u}_{\infty}=0$, the flow is at rest at infinity and in the remaining case, if $\boldsymbol{u}_{\infty} \neq 0$, the flow is past at infinity. In this paper, we are interested in considering the case $\Omega=\mathbb{R}^{3}$ and $\boldsymbol{u}_{\infty} \neq 0$. Our purpose is to study some regularity properties of the weak solutions to the problem $(\mathcal{N S})$. 
This paper is organised as follows: In this section, we recall well-know results about weak solutions, weighted Sobolev spaces and some results of Oseen system in weghted Sobolev spaces. In Section 2, a result about existence of weak solutions for the problem $(\mathcal{N S})$ will be presented. In next sections, we shall obtain some regularity properties of the weak solution $\boldsymbol{u}$ and the associated pressure $\pi$. We shall also consider the identity energy in the last section. In this paper, we use bold type characters to denote vector distributions or spaces of vector distributions with 3 components and $C>0$ usually denotes a generic constant the value of which may change from line to line.

Now we recall the main notations and results, concerning the weighted Sobolev spaces, which we shall use later on.

We define $\mathcal{D}(\Omega)$ to be the linear space of infinite differentiable functions with compact support on $\Omega$. Now, let $\mathcal{D}^{\prime}(\Omega)$ denote the dual space of $\mathcal{D}(\Omega)$, often called the space of distributions on $\Omega$. We denote by $\langle.,$.$\rangle the duality pairing$ between $\mathcal{D}(\Omega)^{\prime}$ and $\mathcal{D}(\Omega)$. Remark that when $\boldsymbol{f}$ is a locally integrable function, then $f$ can be identified with a distribution by

$$
\langle\boldsymbol{f}, \boldsymbol{\varphi}\rangle=\int_{\Omega} \boldsymbol{f}(\boldsymbol{x}) \cdot \boldsymbol{\varphi}(\boldsymbol{x}) d \boldsymbol{x} .
$$

Given a Banach space $B$, with dual space $B^{\prime}$ and a closed subspace $X$ of $B$, we denote by $B^{\prime} \perp X$ (or more simply $X^{\perp}$, if there is no ambiguity as to the duality product) the subspace of $B^{\prime}$ orthogonal to $X$, i.e.

$$
B^{\prime} \perp X=X^{\perp}=\left\{f \in B^{\prime} \mid \forall v \in X,<f, v>=0\right\}=(B / X)^{\prime} .
$$

The space $X^{\perp}$ is also called the polar space of $X$ in $B^{\prime}$. In 1933, Jean Leray [13] who introduced the concept of the weak solution:

Definition 1.1. A weak solution to the problem $(\mathcal{N S})$ is a field $\boldsymbol{u} \in \mathbf{H}_{l o c}^{1}(\bar{\Omega})$ vanishing on $\partial \Omega$, with $\nabla \boldsymbol{u} \in \mathbf{L}^{2}(\Omega)$, div $\boldsymbol{u}=0$ in $\Omega$ and $\lim _{|\boldsymbol{x}| \rightarrow \infty} \int_{S}\left|\boldsymbol{u}\left(\boldsymbol{x}-\boldsymbol{u}_{\infty}\right)\right|=$ $\left|\boldsymbol{u}(\boldsymbol{x})-\boldsymbol{u}_{\infty}\right|=0$ where $S$ is the unit sphere of $\mathbb{R}^{3}$ such that for all $\boldsymbol{\varphi} \in \mathcal{V}(\Omega)=$ $\{\boldsymbol{v} \in \mathcal{D}(\Omega), \operatorname{div} \boldsymbol{v}=0\}:$

$$
\nu \int_{\Omega} \nabla \boldsymbol{u} . \nabla \boldsymbol{\varphi} d \boldsymbol{x}+\int_{\Omega} \boldsymbol{u} . \nabla \boldsymbol{u} \cdot \boldsymbol{\varphi} d \boldsymbol{x}=\langle\boldsymbol{f}, \boldsymbol{\varphi}\rangle .
$$

A typical point in $\mathbb{R}^{3}$ is denoted by $\boldsymbol{x}=\left(x_{1}, x_{2}, x_{3}\right)$ and its norm is given by $|\boldsymbol{x}|=\left(x_{1}^{2}+x_{2}^{2}+x_{3}^{2}\right)^{\frac{1}{2}}$. We define the weight function $\rho(\boldsymbol{x})=\left(1+|\boldsymbol{x}|^{2}\right)^{\frac{1}{2}}$. For each $p \in \mathbb{R}$ and $1<p<\infty$, the conjugate exponent $p^{\prime}$ is given by the relation $\frac{1}{p}+\frac{1}{p^{\prime}}=1$. With $\alpha \in \mathbb{R}$ and $m \in \mathbb{N}$, we set

$$
k=k(m, p, \alpha)= \begin{cases}-1, & \text { if } \frac{3}{p}+\alpha \notin\{1, \ldots, m\}, \\ m-\frac{3}{p}-\alpha, & \text { if } \quad \frac{3}{p}+\alpha \in\{1, \ldots, m\},\end{cases}
$$

and we introduce the definition of the weighted Sobolev spaces.

Definition 1.2. Let $\Omega$ be either an exterior domain or $\Omega=\mathbb{R}^{3}$. Then,

$$
\begin{aligned}
W_{\alpha}^{m, p}(\Omega)=\quad & \left\{u \in \mathcal{D}^{\prime}(\Omega) ; \forall \lambda \in \mathbb{N}^{3},\right. \\
& 0 \leq|\lambda| \leq k, \rho^{\alpha-m+|\lambda|}(\ln (1+\rho))^{-1} \partial^{\lambda} u \in L^{p}(\Omega), \\
& \left.k+1 \leq|\lambda| \leq m, \rho^{\alpha-m+|\lambda|} \partial^{\lambda} u \in L^{p}(\Omega)\right\} .
\end{aligned}
$$


This space is a reflexive Banach space when endowed with the norm:

$$
\begin{aligned}
\|u\|_{W_{\alpha}^{m, p}(\Omega)}=\quad & \left(\sum_{0 \leq|\lambda| \leq k}\left\|\rho^{\alpha-m+|\lambda|}(\ln (1+\rho))^{-1} \partial^{\lambda} u\right\|_{L^{p}(\Omega)}^{p}+\right. \\
& \left.+\sum_{k+1 \leq|\lambda| \leq m}\left\|\rho^{\alpha-m+|\lambda|} \partial^{\lambda} u\right\|_{L^{p}(\Omega)}^{p}\right)^{1 / p} .
\end{aligned}
$$

We also define the semi-norm:

$$
|u|_{W_{\alpha}^{m, p}}(\Omega)=\left(\sum_{|\lambda|=m}\left\|\rho^{\alpha} \partial^{\lambda} u\right\|_{L^{p}(\Omega)}^{p}\right)^{1 / p} .
$$

We note that the logarithmic weight only appears for the case $3 / p+\alpha \in$ $\{1, \ldots, m\}$ and all the local properties of $W_{\alpha}^{m, p}(\Omega)$ coincide with those of the classical Sobolev space $W^{m, p}(\Omega)$. We set $\stackrel{\circ}{W}_{\alpha}^{m, p}(\Omega)=\overline{\mathcal{D}(\Omega)} W_{\alpha}^{m, p}(\Omega)$ and we denote the dual space of $\stackrel{\circ}{W}_{\alpha}^{m, p}(\Omega)$ by $W_{-\alpha}^{-m, p^{\prime}}(\Omega)$, which is the space of distributions. When $\Omega=\mathbb{R}^{3}$, we have $W_{\alpha}^{m, p}\left(\mathbb{R}^{3}\right)=\stackrel{\circ}{W} \underset{\alpha}{m, p}\left(\mathbb{R}^{3}\right)$. If $3 / p+\alpha \notin\{1, \ldots, m\}$, we have the algebraic and topological imbeddings

$$
W_{\alpha}^{m, p}(\Omega) \hookrightarrow W_{\alpha-1}^{m-1, p}(\Omega) \hookrightarrow \ldots \hookrightarrow W_{\alpha-m}^{0, p}(\Omega) .
$$

For all $\lambda \in \mathbb{N}^{n}$ with $|\lambda| \geq 0$, the mapping

$$
u \in W_{\alpha, \beta}^{m, p}(\Omega) \rightarrow \partial^{\lambda} u \in W_{\alpha, \beta}^{m-|\lambda|, p}(\Omega)
$$

is continuous. Moreover, if $\frac{3}{p}+\alpha \notin\{1, \ldots, m\}$, then for any $\gamma$ in $\mathbb{R}$ such that $\frac{3}{p}+\alpha-\gamma \notin\{1, \ldots, m\}$ the mapping $u \rightarrow \rho^{\gamma} u$ is an isomorphism of $W_{\alpha}^{m, p}(\Omega)$ onto $W_{\alpha-\gamma}^{m, p}(\Omega)$. Note that if we only suppose $\frac{3}{p}+\alpha \notin\{1, \ldots, m\}$, the mapping is continuous.

We denote by $[q]$ the integer part of $q$. For any $k \in \mathbb{N}, \mathscr{P}_{k}$ (respectively, $\mathscr{P}_{k}^{\Delta}$ ) stands for the space of polynomials (respectively, harmonic polynomials) of degree $\leq k$. If $k$ is strictly negative integer, we set by convention $\mathscr{P}_{k}=\{0\}$. Let $k$ be an integer, then $\mathscr{P}_{k}$ is included in $W_{\alpha}^{m, p}(\Omega)$ with

$$
k=\left\{\begin{array}{l}
{\left[m-\frac{3}{p}+\alpha\right], \text { if } \frac{3}{p}+\alpha \notin \mathbb{Z}^{-}} \\
m-\frac{3}{p}-\alpha-1, \quad \text { otherwise. }
\end{array}\right.
$$

We introduce the space

$$
\widetilde{W}_{0}^{1, p}(\Omega)=\left\{u \in W_{0}^{1, p}(\Omega), \frac{\partial u}{\partial x_{1}} \in W_{0}^{-1, p}(\Omega)\right\}
$$

which is a Banach space equipped with the following norm

$$
\|u\|_{\widetilde{W}_{0}^{1, p}(\Omega)}=\|u\|_{W_{-1}^{0, p}(\Omega)}+\sum_{i=1}^{3}\left\|\frac{\partial u}{\partial x_{i}}\right\|_{L^{p}(\Omega)}+\left\|\frac{\partial u}{\partial x_{1}}\right\|_{W_{0}^{-1, p}(\Omega)}, \text { if } p \neq 3,
$$




$$
\|u\|_{\widetilde{W}_{0}^{1,3}(\Omega)}=\left\|(\ln (1+\rho))^{-1} u\right\|_{W_{-1}^{0,3}(\Omega)}+\sum_{i=1}^{3}\left\|\frac{\partial u}{\partial x_{i}}\right\|_{L^{3}(\Omega)}+\left\|\frac{\partial u}{\partial x_{1}}\right\|_{W_{0}^{-1,3}(\Omega)},
$$

and $\widetilde{W}_{0}^{-1, p^{\prime}}\left(\mathbb{R}^{3}\right)$ is its dual space. The previous norm is equivalent to the natural one and it allows to prove the density of $\mathcal{D}(\Omega)$ in $\widetilde{W}_{0}^{1, p}(\Omega)$. This result is announced in [7]. We introduce also the space

$$
\mathrm{V}(\Omega)=\left\{\boldsymbol{v} \in \stackrel{\circ}{W}_{0}^{1,2}(\Omega), \operatorname{div} \boldsymbol{v}=0 \text { in } \Omega\right\} .
$$

In order to understand better the the condition $\boldsymbol{u} \rightarrow \boldsymbol{u}_{\infty}$ at infinity of the Navier-Stokes system, we introduce a following lemma (cf [8] ) :

Lemma 1.3. Assume $1<p<3$ and $u \in \mathcal{D}^{\prime}\left(\mathbb{R}^{3}\right)$ such that $\nabla u \in \mathbf{L}^{p}\left(\mathbb{R}^{3}\right)$. Then there exists a unique constant $u_{\infty} \in \mathbb{R}$ such that $u-u_{\infty} \in W_{0}^{1, p}\left(\mathbb{R}^{3}\right)$, where $u_{\infty}$ is defined by

$$
u_{\infty}=\lim _{|x| \rightarrow \infty} \frac{1}{\omega} \int_{S} u(\sigma(|x|)) d \sigma
$$

where $S$ is the unit sphere of $\mathbb{R}^{3}$ and $\omega$ is the area of $S$. Moreover, we have $u-u_{\infty} \in L^{\frac{3 p}{3-p}}\left(\mathbb{R}^{3}\right)$ with the estimate

$$
\begin{gathered}
\left\|u-u_{\infty}\right\|_{L^{\frac{3 p}{3-p}}\left(\mathbb{R}^{3}\right)} \leq C|| \nabla u \|_{\mathbf{L}^{p}\left(\mathbb{R}^{3}\right)}, \\
\lim _{|x| \rightarrow \infty} \int_{S}\left|u(\sigma|x|)-u_{\infty}\right| d \sigma=\lim _{|x| \rightarrow \infty} \int_{S}\left|u(\sigma|x|)-u_{\infty}\right|^{p} d \sigma=0
\end{gathered}
$$

and

$$
\int_{S}\left|u(r \sigma)-u_{\infty}\right|^{p} d \sigma \leq C r^{p-3} \int_{\left\{x \in \mathbb{R}^{3},|x|>r\right\}}|\nabla u|^{p} d x .
$$

Recall also the following Sobolev embeddings

$$
\begin{gathered}
W_{0}^{1, p}\left(\mathbb{R}^{3}\right) \hookrightarrow L^{p *}\left(\mathbb{R}^{3}\right) \text { where } p *=\frac{3 p}{3-p} \text { and } 1<p<3, \\
W_{0}^{1,3}\left(\mathbb{R}^{3}\right) \hookrightarrow V M O\left(\mathbb{R}^{3}\right) \text { where } \operatorname{VMO}\left(\mathbb{R}^{3}\right)=\overline{\mathcal{D}\left(\mathbb{R}^{3}\right)} \|^{\|\cdot\|_{B M O}} .
\end{gathered}
$$

Here, $B M O$ is the space of locally integrable functions in $\mathbb{R}^{3}$ and such that, on all cubes $Q$,

$$
\|f\|_{B M O}=\sup _{Q} \frac{1}{|Q|} \int_{Q}|f(x)-f(Q)| d x<\infty .
$$

Note also that if $\nabla u \in \mathbf{L}^{p}$ with $p>3$ and $u \in L^{r}\left(\mathbb{R}^{3}\right)$ for some $r \geq 1$, then we have $u \in L^{\infty}\left(\mathbb{R}^{3}\right)$.

If $\Omega$ is an exterior domain, we have a corollary as follows:

Corollary 1.4. Let $\Omega \subset \mathbb{R}^{3}$ be an exterior domain. Assume $1<p<3$ and $u \in \mathcal{D}^{\prime}(\Omega)$ such that $\nabla u \in \mathbf{L}^{p}(\Omega)$. Then there exists a unique constant $u_{\infty} \in \mathbb{R}$ such that $u-u_{\infty} \in W_{0}^{1, p}(\Omega)$ and we have the properties (1.1)-(1.3).

Proof. Let $u \in \mathcal{D}^{\prime}(\Omega)$ such that $\nabla u \in \mathbf{L}^{p}(\Omega)$. Then, the restriction of $u$ to $\Omega_{R}$ with a sufficiently large $R$ satisfy $u \in \mathcal{D}^{\prime}\left(\Omega_{R}\right)$ and $\nabla u \in \mathbf{L}^{p}\left(\Omega_{R}\right)$. Therefore, we have $u \in W^{1, p}\left(\Omega_{R}\right)$ and $\left.u\right|_{\partial B_{R}} \in W^{1-1 / p, p}\left(\partial \Omega_{R}\right)$ (see Proposition 2.10 [4]). 
Then there exists $u_{0} \in W^{1, p}\left(\Omega_{R}\right)$ such that $u_{0}=u$ on $\Gamma$ and $u_{0}=0$ on $\partial B_{R}$. We extend $u_{0}$ by zero outside $B_{R}$ and denote $\tilde{u_{0}}$ the extended function that belongs to the classical Sobolev space $W^{1, p}(\Omega)$ and has compact support in $\Omega_{R}$. Note that $v=u-\tilde{u_{0}}$, then $\nabla v \in \mathbf{L}^{p}(\Omega)$ and $v=0$ on $\Gamma$. We set that $\tilde{v}=v$ in $\Omega$ and $\tilde{v}=0$ outside $\Omega$. Then we can deduce that $\nabla \tilde{v} \in \mathbf{L}^{p}\left(\mathbb{R}^{3}\right)$. Therefore there exists a unique constant $u_{\infty}$ such that $\tilde{v}-v_{\infty} \in W_{0}^{1, p}\left(\mathbb{R}^{3}\right)$, or $u-\tilde{u_{0}}-v_{\infty} \in W_{0}^{1, p}\left(\mathbb{R}^{3}\right)$. Then $u-v_{\infty} \in W_{0}^{1, p}(\Omega)$.

Now we shall introduce the following lemma by combining a result of Babenko (1973, Proposition 3) with Theorem II.5.1 [11]. The proof of this lemma can be found in [11].

Lemma 1.5. Let $\Omega \subset \mathbb{R}^{3}$ be a Lipschitz exterior domain or $\Omega=\mathbb{R}^{3}$. Assume that

$$
u \in W_{0}^{1,2}(\Omega) \text { and } \frac{\partial u}{\partial x_{1}} \in L^{q}(\Omega) \text { where } 1<q<2 .
$$

Then $u \in L^{3 q}(\Omega)$ and the following inequality holds:

$$
\|u\|_{L^{3 q}(\Omega)} \leq C\left(\left\|\frac{\partial u}{\partial x_{1}}\right\|_{L^{q}(\Omega)}+\|\nabla u\|_{L^{2}(\Omega)}\right) .
$$

The next lemma gives an another version of this result.

Lemma 1.6. Let $1<p<3$. Assume that $u \in \widetilde{W}_{0}^{1, p}\left(\mathbb{R}^{3}\right)$. Then $u \in L^{\frac{4 p}{4-p}}\left(\mathbb{R}^{3}\right) \cap$ $L^{\frac{3 p}{3-p}}\left(\mathbb{R}^{3}\right)$ and following inequality holds:

$$
\|u\|_{L^{\frac{4 p}{4-p}\left(\mathbb{R}^{3}\right)}}+\|u\|_{L^{\frac{3 p}{3-p}}\left(\mathbb{R}^{3}\right)} \leq C\|u\|_{\widetilde{W}_{0}^{1, p}\left(\mathbb{R}^{3}\right)^{\prime}} .
$$

Proof. We already showed that if $u \in W_{0}^{1, p}\left(\mathbb{R}^{3}\right)$ with $1<p<3$, then $u \in$ $L^{\frac{3 p}{3-p}}\left(\mathbb{R}^{3}\right)$ satisfying

$$
\|u\|_{L^{\frac{3 p}{3-p}\left(\mathbb{R}^{3}\right)}} \leq C|| \nabla u \|_{L^{p}\left(\mathbb{R}^{3}\right)} .
$$

We know that $\mathcal{D}\left(\mathbb{R}^{3}\right)$ is dense in $W_{0}^{1, p}\left(\mathbb{R}^{3}\right)$, then there exists a sequence $\left(\varphi_{k}\right)_{k \in \mathbb{N}} \in$ $\mathcal{D}\left(\mathbb{R}^{3}\right)$ which converges towards 1 in $W_{0}^{1, p^{\prime}}\left(\mathbb{R}^{3}\right)$. By hypothesis, we deduce $\Delta u \in W_{0}^{-1, p}\left(\mathbb{R}^{3}\right)$. Then, we have

$$
\begin{aligned}
\langle\Delta u, 1\rangle_{W_{0}^{-1, p}\left(\mathbb{R}^{3}\right) \times W_{0}^{1, p^{\prime}}\left(\mathbb{R}^{3}\right)} & =\lim _{k \rightarrow+\infty}\left\langle\Delta u, \varphi_{k}\right\rangle_{W_{0}^{-1, p}\left(\mathbb{R}^{3}\right) \times W_{0}^{1, p^{\prime}}\left(\mathbb{R}^{3}\right)} \\
& =-\lim _{k \rightarrow+\infty}\left\langle\nabla u, \nabla \varphi_{k}\right\rangle_{L^{p}\left(\mathbb{R}^{3}\right) \times L^{p^{\prime}}\left(\mathbb{R}^{3}\right)}=0 .
\end{aligned}
$$

Analogously, since $\mathcal{D}\left(\mathbb{R}^{3}\right)$ is dense in $\widetilde{W}_{0}^{1, p}\left(\mathbb{R}^{3}\right)$ (see [7]), then we can deduce that

$$
\left\langle\frac{\partial u}{\partial x_{1}}, 1\right\rangle_{W_{0}^{-1, p}\left(\mathbb{R}^{3}\right) \times W_{0}^{1, p^{\prime}}\left(\mathbb{R}^{3}\right)}=0 .
$$

We set

$$
-\Delta u+\frac{\partial u}{\partial x_{1}}=f
$$

Then by hypothesis and [5], we have $f \in W_{0}^{-1, p}\left(\mathbb{R}^{3}\right)$ satisfying the compatibility condition as follows

$$
\langle f, 1\rangle_{W_{0}^{-1, p}\left(\mathbb{R}^{3}\right) \times W_{0}^{1, p}\left(\mathbb{R}^{3}\right)}=0 .
$$


Then, from [8], the equation as follows

$$
-\Delta w+\frac{\partial w}{\partial x_{1}}=f \text { in } \mathbb{R}^{3}
$$

has a unique solution $w \in L^{\frac{3 p}{3-p}}\left(\mathbb{R}^{3}\right) \cap L^{\frac{4 p}{4-p}}\left(\mathbb{R}^{3}\right)$ such that $\nabla w \in \mathbf{L}^{p}\left(\mathbb{R}^{3}\right)$, $\frac{\partial w}{\partial x_{1}} \in W_{0}^{-1, p}\left(\mathbb{R}^{3}\right)$ also satisfying

$$
\begin{aligned}
& \|w\|_{L^{\frac{3 p}{3-p}}\left(\mathbb{R}^{3}\right)}+\|w\|_{L^{\frac{4 p}{4-p}\left(\mathbb{R}^{3}\right)}}+\|\nabla w\|_{\mathbf{L}^{p}\left(\mathbb{R}^{3}\right)}+\left\|\frac{\partial w}{\partial x_{1}}\right\|_{W_{0}^{-1, p}\left(\mathbb{R}^{3}\right)} \\
& \leq C\|f\|_{W_{0}^{-1, p}\left(\mathbb{R}^{3}\right)} .
\end{aligned}
$$

We set $z=u-w$. Subtracting (1.5) to (1.6), we get $-\Delta z+\frac{\partial z}{\partial x_{1}}=0$ in $\mathbb{R}^{3}$. Since $z \in L^{3 p /(3-p)}\left(\mathbb{R}^{3}\right)$, then, from Lemma 4.1 [8], we deduce that $z$ is a polynomial and then $z=0$. From (1.7), we have (1.4). The proof is complete.

Analogously as in Lemma 1.6, it is easy to deduce the following.

Lemma 1.7. Let $1<p<2$. Assume that $u \in W_{0}^{2, p}\left(\mathbb{R}^{3}\right)$ and $\frac{\partial u}{\partial x_{1}} \in L^{p}\left(\mathbb{R}^{3}\right)$. Then we have $u \in L^{\frac{2 p}{2-p}}\left(\mathbb{R}^{3}\right) \cap L^{\frac{3 p}{3-2 p}}\left(\mathbb{R}^{3}\right)$ if $1<p<3 / 2$ and $u \in L^{s}\left(\mathbb{R}^{3}\right)$ for all $s \geq \frac{2 p}{2-p}$ if $3 / 2 \leq p<2$.

Definition 1.8. Let $1<p<\infty$. Let $\gamma, \delta \in \mathbb{R}$ be such that $\gamma \in[3,4], \gamma>p$, $\delta \in\left[\frac{3}{2}, 2\right], \delta>p$. We define two reals $r=r(p, \gamma)$ and $s=s(p, \delta)$ as follow

$$
\frac{1}{r}=\frac{1}{p}-\frac{1}{\gamma} \text { and } \quad \frac{1}{s}=\frac{1}{p}-\frac{1}{\delta} .
$$

Remark 1.9. From Definition 1.8, we can deduce that

i) If $1<p<3$, then $\frac{4 p}{4-p} \leq r \leq \frac{3 p}{3-p}$,

ii) If $3 \leq p<4$, then $\frac{4 p}{4-p} \leq r<\infty$,

iii) If $1<p<3 / 2$, then $\frac{2 p}{2-p} \leq s \leq \frac{3 p}{3-2 p}$,

iv) If $3 / 2 \leq p<2$, then $\frac{2 p}{2-p} \leq s<\infty$.

Finally, we introduce the properties concerning the Oseen equations which will be useful in the next parts. We consider the non homogeneous Oseen problem : given a vector field $\boldsymbol{f}$ and a function $g$, we look for a solution $(\boldsymbol{u}, \pi)$ to the system

$$
(\mathcal{O S}) \begin{cases}-\Delta \boldsymbol{u}+\frac{\partial \boldsymbol{u}}{\partial x_{1}}+\nabla \pi=f & \text { in } \mathbb{R}^{3}, \\ \operatorname{div} \boldsymbol{u}=g & \text { in } \mathbb{R}^{3} .\end{cases}
$$

Theorem 1.10. [7] Let $r$ and $s$ be the numbers given in Definition 1.8. Assume $(\boldsymbol{f}, g) \in \mathbf{L}^{p}\left(\mathbb{R}^{3}\right) \times \widetilde{W}_{0}^{1, p}\left(\mathbb{R}^{3}\right)$.

(i) If $1<p<2$, then Problem $(\mathcal{O S})$ has a unique solution $(\boldsymbol{u}, \pi) \in \mathbf{L}^{s}\left(\mathbb{R}^{3}\right) \times$ 
$W_{0}^{1, p}\left(\mathbb{R}^{3}\right)$ such that $\nabla \boldsymbol{u} \in \mathbf{L}^{r}\left(\mathbb{R}^{3}\right), \nabla^{2} \boldsymbol{u} \in \mathbf{L}^{p}\left(\mathbb{R}^{3}\right)$ and $\frac{\partial \boldsymbol{u}}{\partial x_{1}} \in \mathbf{L}^{p}\left(\mathbb{R}^{3}\right)$. Moreover, the following estimate holds

$$
\begin{array}{r}
\|\boldsymbol{u}\|_{\mathbf{L}^{s}\left(\mathbb{R}^{3}\right)}+\|\nabla \boldsymbol{u}\|_{\mathbf{L}^{r}\left(\mathbb{R}^{3}\right)}+\left\|\nabla^{2} \boldsymbol{u}\right\|_{\mathbf{L}^{p}\left(\mathbb{R}^{3}\right)} \\
+\left\|\frac{\partial \boldsymbol{u}}{\partial x_{1}}\right\|_{\mathbf{L}^{p}\left(\mathbb{R}^{3}\right)}+\|\pi\|_{W_{0}^{1, p}\left(\mathbb{R}^{3}\right)} \\
\leq C\left(\|\boldsymbol{f}\|_{\mathbf{L}^{p}\left(\mathbb{R}^{3}\right)}+\|g\|_{\widetilde{W}_{0}^{1, p}\left(\mathbb{R}^{3}\right)}\right) .
\end{array}
$$

(ii) If $2 \leq p<3$, then Problem $(\mathcal{O S})$ has a solution $(\boldsymbol{u}, \pi) \in \mathbf{W}_{0}^{1, r}\left(\mathbb{R}^{3}\right) \times$ $W_{0}^{1, p}\left(\mathbb{R}^{3}\right)$, unique up to an element of $\mathcal{N}_{0}$, such that $\nabla^{2} \boldsymbol{u} \in \mathbf{L}^{p}\left(\mathbb{R}^{3}\right)$ and $\frac{\partial \boldsymbol{u}}{\partial x_{1}} \in$ $\mathbf{L}^{p}\left(\mathbb{R}^{3}\right)$ also satisfying

$$
\begin{array}{r}
\inf _{K \in \mathbb{R}^{3}}\|\boldsymbol{u}+\mathbf{K}\|_{\mathbf{W}_{0}^{1, r}\left(\mathbb{R}^{3}\right)}+\left\|\nabla^{2} \boldsymbol{u}\right\|_{\mathbf{L}^{p}\left(\mathbb{R}^{3}\right)}+\left\|\frac{\partial \boldsymbol{u}}{\partial x_{1}}\right\|_{\mathbf{L}^{p}\left(\mathbb{R}^{3}\right)}+\|\pi\|_{W_{0}^{1, p}\left(\mathbb{R}^{3}\right)} \\
\leq C\left(\|\boldsymbol{f}\|_{\mathbf{L}^{p}\left(\mathbb{R}^{3}\right)}+\|g\|_{\widetilde{W}_{0}^{1, p}\left(\mathbb{R}^{3}\right)}\right) .
\end{array}
$$

(iii) If $p \geq 3$, then Problem $(\mathcal{O S})$ has a solution $(\boldsymbol{u}, \pi) \in \mathbf{W}_{0}^{2, r}\left(\mathbb{R}^{3}\right) \times W_{0}^{1, p}\left(\mathbb{R}^{3}\right)$, unique up to an element of $\mathcal{N}_{1}$, such that $\frac{\partial \boldsymbol{u}}{\partial x_{1}} \in \mathbf{L}^{p}\left(\mathbb{R}^{3}\right)$. Moreover, we have

$$
\begin{array}{r}
\inf _{(\boldsymbol{\lambda}, \mu) \in \mathcal{N}_{1}}\left(\|\boldsymbol{u}+\boldsymbol{\lambda}\|_{\mathbf{W}_{0}^{2, p}\left(\mathbb{R}^{3}\right)}+\right. \\
\leq C\left(\|\boldsymbol{\pi}+\mu\|_{W_{0}^{1, p}\left(\mathbb{R}^{3}\right)}\right)+\left\|\frac{\partial \boldsymbol{u}}{\partial x_{1}}\right\|_{\mathbf{L}^{p}\left(\mathbb{R}^{3}\right)} \\
\left.\left.\leq C \mathbb{R}^{3}\right)+\|g\|_{\widetilde{W}_{0}^{1, p}\left(\mathbb{R}^{3}\right)}\right) .
\end{array}
$$

Theorem 1.11. [7] Let $r$ be the number given in Definition 1.8. Assume that $\boldsymbol{f} \in \mathbf{W}_{0}^{-1, p}\left(\mathbb{R}^{3}\right)$ and satisfies the compatibility condition

$$
\forall \boldsymbol{\lambda} \in \mathscr{P}_{\left[1-3 / p^{\prime}\right]},\langle\boldsymbol{f}, \boldsymbol{\lambda}\rangle_{\mathbf{W}_{0}^{-1, p}\left(\mathbb{R}^{3}\right) \times \mathbf{W}_{0}^{1, p^{\prime}}\left(\mathbb{R}^{3}\right)}=0 .
$$

Let $g \in L^{p}\left(\mathbb{R}^{3}\right)$ such that $\frac{\partial g}{\partial x_{1}} \in W_{0}^{-2, p}\left(\mathbb{R}^{3}\right)$, satisfies the compatibility condition

$$
\forall \lambda \in \mathscr{P}_{\left[2-3 / p^{\prime}\right]},\left\langle\frac{\partial g}{\partial x_{1}}, \lambda\right\rangle_{W_{0}^{-2, p}\left(\mathbb{R}^{3}\right) \times W_{0}^{2, p^{\prime}}\left(\mathbb{R}^{3}\right)}=0 .
$$

(i) If $1<p<4$, then the Oseen system $(\mathcal{O S})$ has a unique solution $(\boldsymbol{u}, \pi) \in$ $\mathbf{L}^{r}\left(\mathbb{R}^{3}\right) \times L^{p}\left(\mathbb{R}^{3}\right)$ such that $\nabla \boldsymbol{u} \in \mathbf{L}^{p}\left(\mathbb{R}^{3}\right)$ and $\frac{\partial \boldsymbol{u}}{\partial x_{1}} \in \mathbf{W}_{0}^{-1, p}\left(\mathbb{R}^{3}\right)$. Moreover, the following estimate holds

$$
\begin{aligned}
& \|\boldsymbol{u}\|_{\mathbf{L}^{r}\left(\mathbb{R}^{3}\right)}+\|\nabla \boldsymbol{u}\|_{\mathbf{L}^{p}\left(\mathbb{R}^{3}\right)}+\left\|\frac{\partial \boldsymbol{u}}{\partial x_{1}}\right\|_{\mathbf{W}_{0}^{-1, p}\left(\mathbb{R}^{3}\right)}+\|\pi\|_{L^{p}\left(\mathbb{R}^{3}\right)} \\
& \leq C\left(\|\boldsymbol{f}\|_{\mathbf{W}_{0}^{-1, p}\left(\mathbb{R}^{3}\right)}+\|g\|_{L^{p}\left(\mathbb{R}^{3}\right)}+\left\|\frac{\partial g}{\partial x_{1}}\right\|_{W_{0}^{-2, p}\left(\mathbb{R}^{3}\right)}\right) .
\end{aligned}
$$

(ii) If $p \geq 4$, then the Oseen system $(\mathcal{O S})$ has a unique solution $(\boldsymbol{u}, \pi) \in$ $\widetilde{\mathbf{W}}_{0}^{1, p}\left(\mathbb{R}^{3}\right) \times L^{p}\left(\mathbb{R}^{3}\right)$, unique up to an element of $\mathcal{N}_{0}$. Moreover, the following estimate holds

$$
\begin{array}{r}
\inf _{\mathbf{K} \in \mathbb{R}^{3}}\|\boldsymbol{u}+\mathbf{K}\|_{\widetilde{\mathbf{W}}_{0}^{1, p}\left(\mathbb{R}^{3}\right)}+\|\pi\|_{L^{p}\left(\mathbb{R}^{3}\right)} \\
\leq C\left(\|\boldsymbol{f}\|_{\mathbf{W}_{0}^{-1, p}\left(\mathbb{R}^{3}\right)}+\|g\|_{L^{p}\left(\mathbb{R}^{3}\right)}+\left\|\frac{\partial g}{\partial x_{1}}\right\|_{W_{0}^{-2, p}\left(\mathbb{R}^{3}\right)}\right) .
\end{array}
$$




\section{Existence of weak solutions in weighted Sobolev spaces}

We shall consider the Navier-Stokes problem in $\mathbb{R}^{3}$ :

$$
(\mathcal{N S}) \begin{cases}-\nu \Delta \boldsymbol{u}+\boldsymbol{u} \cdot \nabla \boldsymbol{u}+\nabla \pi=f & \text { in } \mathbb{R}^{3}, \\ \operatorname{div} \boldsymbol{u}=0 & \text { in } \mathbb{R}^{3}, \\ \boldsymbol{u} \longrightarrow \boldsymbol{u}_{\infty} & \text { if }|x| \rightarrow \infty\end{cases}
$$

where $\boldsymbol{u}_{\infty}$ is a constant vector in $\mathbb{R}^{3}$. Without loss of generality, we can set $\boldsymbol{u}_{\infty}=\lambda \boldsymbol{e}_{1}$ with $\boldsymbol{e}_{1}=(1,0,0)$ and $\lambda \geq 0$. From now on, we consider the case of a fixed $\lambda>0$. First, we prove the existence of weak solutions and then, we shall the regularity of these solutions in dimention 3 . We consider the following lemma.

Lemma 2.1. If $f \in W_{0}^{-1,2}\left(\mathbb{R}^{3}\right)$, then there exists $\boldsymbol{F} \in \mathbf{L}^{2}\left(\mathbb{R}^{3}\right)$ such that $f=$ $\operatorname{div} \boldsymbol{F}$ in $\mathbb{R}^{3}$ with the estimate

$$
\|\boldsymbol{F}\|_{\mathbf{L}^{2}\left(\mathbb{R}^{3}\right)} \leq C|| f \|_{W_{0}^{-1,2}\left(\mathbb{R}^{3}\right)} .
$$

Additionally suppose that $f \in W_{0}^{-1, p}\left(\mathbb{R}^{3}\right)$, and furthermore assume that $\langle f, 1\rangle=$ 0 if $p \leq \frac{3}{2}$, then $\boldsymbol{F} \in \mathbf{L}^{p}\left(\mathbb{R}^{3}\right)$ and we have the estimate

$$
\|\boldsymbol{F}\|_{\mathbf{L}^{p}\left(\mathbb{R}^{3}\right)} \leq C^{\prime}|| f \|_{W_{0}^{-1, p}\left(\mathbb{R}^{3}\right)} .
$$

Proof. If $f \in W_{0}^{-1,2}\left(\mathbb{R}^{3}\right)$, from Theorem 9.5 [5], there exists a unique $z \in$ $W_{0}^{1,2}\left(\mathbb{R}^{3}\right)$ such that $\Delta z=f$ in $\mathbb{R}^{3}$ and

$$
\|z\|_{W_{0}^{1,2}\left(\mathbb{R}^{3}\right)} \leq C|| f \|_{W_{0}^{-1,2}\left(\mathbb{R}^{3}\right)} .
$$

We set that $\boldsymbol{F}=\nabla z$, but $z \in W_{0}^{1,2}\left(\mathbb{R}^{3}\right)$, from Proposition 9.2 [5], we have $\boldsymbol{F} \in \mathbf{L}^{2}\left(\mathbb{R}^{3}\right)$ and (2.1). Moreover, if $f \in W_{0}^{-1, p}\left(\mathbb{R}^{3}\right)$ then there exists a unique $h \in W_{0}^{1, p}\left(\mathbb{R}^{3}\right) / \mathscr{P}_{\left[1-\frac{3}{p}\right]}$ such that $f=\Delta h$ in $\mathbb{R}^{3}$ and

$$
\|h\|_{W_{0}^{1, p}\left(\mathbb{R}^{3}\right) / \mathscr{P}_{\left[1-\frac{3}{p}\right]}} \leq C^{\prime}\|f\|_{W_{0}^{-1, p}\left(\mathbb{R}^{3}\right)} .
$$

Then $\nabla(z-h)$ is harmonic in $\mathbf{L}^{2}\left(\mathbb{R}^{3}\right)+\mathbf{L}^{p}\left(\mathbb{R}^{3}\right)$ and consequently, $\nabla z=\nabla h$ and $\boldsymbol{F} \in \mathbf{L}^{2}\left(\mathbb{R}^{3}\right) \cap \mathbf{L}^{p}\left(\mathbb{R}^{3}\right)$ with the estimate (2.2).

We now return to the question of the existence of weak solutions of the Navier-Stokes Equations in $\mathbb{R}^{3}$. The next theorem is well known, then we give here a sketch of the proof.

Theorem 2.2. Given a force $\boldsymbol{f} \in \mathbf{W}_{0}^{-1,2}\left(\mathbb{R}^{3}\right)$, the problem $(\mathcal{N S})$ has a weak solution $\boldsymbol{u}$ satisfying $\boldsymbol{u}-\boldsymbol{u}_{\infty} \in \mathbf{W}_{0}^{1,2}\left(\mathbb{R}^{3}\right)$ and there exists a function $\pi \in L_{\text {loc }}^{2}\left(\mathbb{R}^{3}\right)$, unique up to a constant, such that $(\boldsymbol{u}, \pi)$ solves the problem $(\mathcal{N S})$ in the sense of distributions and we have the following estimation

$$
\left\|\boldsymbol{u}-\boldsymbol{u}_{\infty}\right\|_{\mathbf{W}_{0}^{1,2}\left(\mathbb{R}^{3}\right)} \leq C\|\boldsymbol{f}\|_{\mathbf{W}_{0}^{-1,2}\left(\mathbb{R}^{3}\right)} .
$$


Proof. Thanks to Lemma 2.1, for each $i=1,2,3$, we know that there exists $\boldsymbol{F}_{i} \in L^{2}\left(\mathbb{R}^{3}\right)^{3}$ such that $f_{i}=\operatorname{div} \boldsymbol{F}_{i} \in W_{0}^{-1,2}\left(\mathbb{R}^{3}\right)$ with the estimation (2.1). We consider the following approximating problems (for each $m \in \mathbb{N}^{*}$ ):

$$
\begin{aligned}
-\nu \Delta \boldsymbol{u}^{m}+\boldsymbol{u}^{m} \cdot \nabla \boldsymbol{u}^{m}+\nabla \pi^{m}=\operatorname{div} \boldsymbol{F} & \text { in } B_{R_{m}}=B_{m}, \\
\operatorname{div} \boldsymbol{u}^{m}=0 & \text { in } B_{m}, \\
\boldsymbol{u}^{m}=\boldsymbol{u}_{\infty} & \text { on } \partial B_{m}
\end{aligned}
$$

where $B_{m}$ is the open ball of radius $R_{m}>0$ centered at the origin. We know that there exists a weak solution $\left(\boldsymbol{u}^{m}, \pi^{m}\right) \in \mathbf{H}^{1}\left(B_{m}\right) \times L^{2}\left(B_{m}\right)$ of (2.4) satisfying the following estimation:

$$
\nu\left\|\nabla \boldsymbol{u}^{m}\right\|_{\mathbf{L}^{2}\left(B_{m}\right)} \leq\|\boldsymbol{F}\|_{\mathbf{L}^{2}\left(B_{m}\right)} \leq\|\boldsymbol{f}\|_{\mathbf{W}_{0}^{-1,2}\left(\mathbb{R}^{3}\right)} .
$$

We extend $\boldsymbol{u}^{m}$ by $\boldsymbol{u}_{\infty}$ outside $B_{m}$ and we denote the extended function by $\tilde{\boldsymbol{u}}^{m}$. We set $\tilde{\boldsymbol{v}}^{m}=\tilde{\boldsymbol{u}}^{m}-\boldsymbol{u}_{\infty}$. Since $\tilde{\boldsymbol{v}}^{m} \in \mathbf{W}_{0}^{1,2}\left(\mathbb{R}^{3}\right)$, then we deduce from Lemma 1.3 that

$$
\left\|\widetilde{\boldsymbol{u}^{m}}-\boldsymbol{u}_{\infty}\right\|_{\mathbf{L}^{6}\left(\mathbb{R}^{3}\right)}+\left\|\nabla \widetilde{\boldsymbol{u}^{m}}\right\|_{\mathbf{L}^{2}\left(\mathbb{R}^{3}\right)} \leq C\|\boldsymbol{f}\|_{\mathbf{W}_{0}^{-1,2}\left(\mathbb{R}^{3}\right)} .
$$

Thus there exists a subsequence of $\left(\widetilde{\boldsymbol{u}^{m}}\right)$ and $\boldsymbol{u}$ such that $\boldsymbol{u}-\boldsymbol{u}_{\infty} \in \mathbf{W}_{0}^{1,2}\left(\mathbb{R}^{3}\right)$ and such that $\widetilde{\boldsymbol{v}^{m}}=\widetilde{\boldsymbol{u}^{m}}-\boldsymbol{u}_{\infty} \rightarrow \boldsymbol{u}-\boldsymbol{u}_{\infty}=\boldsymbol{v}$ in $\mathbf{L}^{6}\left(\mathbb{R}^{3}\right)$ and $\nabla \widetilde{\boldsymbol{u}^{m}} \rightarrow \nabla \boldsymbol{u}$ in $\mathbf{L}^{2}\left(\mathbb{R}^{3}\right)$. Moreover, we have

$$
\nu\|\nabla \boldsymbol{u}\|_{\mathbf{L}^{2}\left(\mathbb{R}^{3}\right)} \leq \liminf \nu\left\|\nabla \tilde{\boldsymbol{u}^{m}}\right\|_{\mathbf{L}^{2}\left(\mathbb{R}^{3}\right)} \leq\|\boldsymbol{f}\|_{\mathbf{W}_{0}^{-1,2}\left(\mathbb{R}^{3}\right)}
$$

and (2.3) is satisfied.

Let us now check that $\boldsymbol{u}$ is a weak solution. Let $\varphi \in \mathcal{V}\left(\mathbb{R}^{3}\right)$ and $N>0$ be an integer such that $\operatorname{supp} \varphi \subset B_{N}$. Then, for all $m \geq N$, we deduce from (2.4) that

$$
\nu \int_{\mathbb{R}^{3}} \nabla \widetilde{\boldsymbol{u}^{m}} \cdot \nabla \boldsymbol{\varphi} d \boldsymbol{x}+\int_{\mathbb{R}^{3}} \tilde{\boldsymbol{u}}^{m} \cdot \nabla \widetilde{\boldsymbol{u}^{m}} \cdot \boldsymbol{\varphi} d \boldsymbol{x}=\langle\boldsymbol{f}, \boldsymbol{\varphi}\rangle .
$$

In view of (2.5), we can pass to limit in the first integral. We know that the imbedding $\mathbf{H}^{1}\left(B_{N}\right) \subset \mathbf{L}^{2}\left(B_{N}\right)$ is compact, then $\tilde{\boldsymbol{u}}^{m}$ converges strongly to $\boldsymbol{u}$ in $\mathbf{L}^{2}\left(B_{N}\right)$. Then, this convergence together with (2.5) ensures the convergence of the second integral of (2.6), then we have $\boldsymbol{u}$ is a weak solution of $(\mathcal{N S})$.

Finally, the existence of a pressure $\pi \in \mathcal{D}^{\prime}\left(\mathbb{R}^{3}\right)$ such that $(\boldsymbol{u}, \pi)$ satisfies $(\mathcal{N S})$ in the sense of distributions follows from the Definition 1.1 and from a well-known consequence of a very general theorem of G.de Rham. It is easy to that $\boldsymbol{f}-\boldsymbol{u} . \nabla \boldsymbol{u}+\nu \Delta \boldsymbol{u} \in \mathbf{H}_{l o c}^{-1}\left(\mathbb{R}^{3}\right)$ which implies that $\pi \in L_{l o c}^{2}\left(\mathbb{R}^{3}\right)$.

In Theorem 2.2, we see that a pressure $\pi$ locally belongs to $L^{2}$. At the beginning, we shall establish, without additional assumption, of the properties of integrability at infinity of the pressure.

Proposition 2.3. Let $\boldsymbol{f} \in \mathbf{W}_{0}^{-1,2}\left(\mathbb{R}^{3}\right)$. The pressure $\pi$ obtained in Theorem 2.2 has a representative such that

$$
\pi=\tau^{1}+\tau^{2} \text { with } \tau^{1} \in L^{2}\left(\mathbb{R}^{3}\right) \text { and } \tau^{2} \in W_{0}^{1,3 / 2}\left(\mathbb{R}^{3}\right) .
$$


Proof. Let $R_{1}$ and $R_{2}$ be reals such that $R_{2}>R_{1}>0$ and choose some functions $\psi_{1}$ and $\psi_{2}$ such that

$$
\begin{gathered}
\psi_{1} \in C^{\infty}\left(\mathbb{R}^{3}\right), \psi_{1}(\boldsymbol{x})=0 \text { if }|\boldsymbol{x}| \leq R_{1}, \psi_{1}(\boldsymbol{x})=1 \text { if }|\boldsymbol{x}| \geq R_{2}, \\
\forall \boldsymbol{x} \in \mathbb{R}^{3}, \psi_{1}(\boldsymbol{x})+\psi_{2}(\boldsymbol{x})=1 .
\end{gathered}
$$

Let $\boldsymbol{v}=\boldsymbol{u}-\boldsymbol{u}_{\infty}$ where $\boldsymbol{u}$ is a solution given by Theorem 2.2 and let $\pi \in L_{l o c}^{2}\left(\mathbb{R}^{3}\right)$ be the associated pressure. We define $\left(\boldsymbol{v}^{1}, \pi^{1}\right)$ as follows

$$
\left(\boldsymbol{v}^{1}, \pi^{1}\right)=\left(\boldsymbol{v} \psi_{1}, \pi \psi_{1}\right) \text { in } \mathbb{R}^{3}, \quad\left(\boldsymbol{v}^{1}, \pi^{1}\right)=(\mathbf{0}, 0) \text { in } B_{1},
$$

where $B_{1}$ is the open ball of radius $R_{1}$ and set $\left(\boldsymbol{v}^{2}, \pi^{2}\right)=\left(\boldsymbol{v} \psi_{2}, \pi \psi_{2}\right)$ in $\mathbb{R}^{3}$. Then $\left(\boldsymbol{v}^{i}, \pi^{i}\right)(i=1,2)$, satisfies

$$
-\nu \Delta \boldsymbol{v}^{i}+\lambda \frac{\partial \boldsymbol{v}^{i}}{\partial x_{1}}+\nabla \pi^{i}=\boldsymbol{f}^{i} \text { and } \operatorname{div} \boldsymbol{v}^{i}=g^{i} \text { in } \mathbb{R}^{3},
$$

where $\boldsymbol{f}^{i}=\left[\boldsymbol{f} \psi_{i}-\nu \boldsymbol{v} \Delta \psi_{i}-2 \nu \nabla \boldsymbol{v} \nabla \psi_{i}+\pi \nabla \psi_{i}\right]+\left[\lambda \boldsymbol{v} \frac{\partial \psi_{i}}{\partial x_{1}}-(\boldsymbol{v} . \nabla \boldsymbol{v}) \psi_{i}\right]:=k_{i}+h_{i}$ and $g^{i}=-\boldsymbol{v} \cdot \nabla \psi_{i}$. We have $\pi=\pi^{1}+\pi^{2}$ and from Theorem 2.2, we obtain $\pi^{2} \in L^{2}\left(\mathbb{R}^{3}\right)$. Thus, the main of the proof deals with the properties of $\pi^{1}$ and therefore of $\left(f^{1}, g^{1}\right)$. We consider

$$
-\nu \Delta \boldsymbol{a}^{1}+\lambda \frac{\partial \boldsymbol{a}^{1}}{\partial x_{1}}+\nabla b^{1}=k_{1} \text { and } \operatorname{div} \boldsymbol{a}^{1}=-\boldsymbol{v} \nabla \psi_{1} \text { in } \mathbb{R}^{3} .
$$

Since $\psi_{1}$ is bounded and has bounded derivatives with compact support, it is easy to check that the term $\boldsymbol{f} \psi_{1}, \boldsymbol{v} \Delta \psi_{1}, \nabla \boldsymbol{v} \nabla \psi_{1}$ and $\pi \nabla \psi_{1}$ belong to $\mathbf{W}_{0}^{-1,2}\left(\mathbb{R}^{3}\right)$ and because $\mathbf{W}_{0}^{1,2}\left(\mathbb{R}^{3}\right) \subset \mathbf{L}^{6}\left(\mathbb{R}^{3}\right)$ then we have $\boldsymbol{v} \cdot \frac{\partial \psi_{1}}{\partial x_{1}} \in \mathbf{L}^{q}\left(\mathbb{R}^{3}\right)$ for all $q \in[1,6]$. Even simple is to prove that $g^{1}=-\boldsymbol{v} . \nabla \psi_{1} \in L^{2}\left(\mathbb{R}^{3}\right) \cap W_{0}^{-1,2}\left(\mathbb{R}^{3}\right)$ and therefore $\frac{\partial g^{1}}{\partial x_{1}} \in W_{0}^{-2,2}\left(\mathbb{R}^{3}\right)$ satisfying the following compatibility condition

$$
\left\langle\frac{\partial g^{1}}{\partial x_{1}}, 1\right\rangle_{W_{0}^{-2,2}\left(\mathbb{R}^{3}\right) \times W_{0}^{2,2}\left(\mathbb{R}^{3}\right)}=0 .
$$

Applying Theorem 1.11, there exists a unique solution $\left(\boldsymbol{a}^{1}, b^{1}\right) \in\left(\widetilde{\mathbf{W}}_{0}^{1,2}\left(\mathbb{R}^{3}\right) \times\right.$ $\left.L^{2}\left(\mathbb{R}^{3}\right)\right)$ of (2.8) such that $\boldsymbol{a}^{1} \in \mathbf{L}^{r_{1}}\left(\mathbb{R}^{3}\right)$ where $4 \leq r_{1} \leq 6$. Thanks to Hölder inequality, we deduce that $(\boldsymbol{v} \cdot \nabla \boldsymbol{v}) \psi_{1} \in \mathbf{L}^{3 / 2}\left(\mathbb{R}^{3}\right)$ and, in particular, we have $\boldsymbol{v} \cdot \frac{\partial \psi_{1}}{\partial x_{1}} \in \mathbf{L}^{3 / 2}\left(\mathbb{R}^{3}\right)$. Therefore, from Theorem 1.10, the system as follows

$$
-\nu \Delta \boldsymbol{a}^{2}+\lambda \frac{\partial \boldsymbol{a}^{2}}{\partial x_{1}}+\nabla b^{2}=h_{1} \text { and } \operatorname{div} \boldsymbol{a}^{2}=0 \text { in } \mathbb{R}^{3},
$$

has a unique solution $\left(\boldsymbol{a}^{2}, b^{2}\right) \in \mathbf{L}^{s_{1}}\left(\mathbb{R}^{3}\right) \times W_{0}^{1,3 / 2}\left(\mathbb{R}^{3}\right)$ such that $\nabla \boldsymbol{a}^{2} \in \mathbf{L}^{r_{2}}\left(\mathbb{R}^{3}\right)$, $\nabla^{2} \boldsymbol{a}^{2} \in \mathbf{L}^{3 / 2}\left(\mathbb{R}^{3}\right)$ and $\frac{\partial \boldsymbol{a}^{2}}{\partial x_{1}} \in \mathbf{L}^{3 / 2}\left(\mathbb{R}^{3}\right)$ for all $s_{1} \in[6, \infty)$ and $r_{2} \in[12 / 5,3]$.

We set $\boldsymbol{z}=\boldsymbol{v}^{1}-\boldsymbol{a}^{1}-\boldsymbol{a}^{2}$ and $\theta=\pi^{1}-b^{1}-b^{2}$. Subtracting (2.7) to (2.8) and (2.9), we get

$$
-\nu \Delta \boldsymbol{z}+\lambda \frac{\partial \boldsymbol{z}}{\partial x_{1}}+\nabla \theta=\mathbf{0} \text { and } \quad \operatorname{div} \boldsymbol{z}=0 \text { in } \mathbb{R}^{3} .
$$


Proceeding as in the proof of Theorem 3.1 part a) in the next section, we can deduce that $\boldsymbol{z}=0$, then $\nabla \theta=0$, and by the way the existence of a constant $c$ such that $\pi^{1}=b^{1}+b^{2}+c$. Therefore, the proposition is proved setting $\tau^{1}=\pi^{2}+b^{1}, \tau^{2}=b^{2}$.

\section{Regularity of the weak solutions}

Let $\boldsymbol{v}=\boldsymbol{u}-\boldsymbol{u}_{\infty}$ where $\boldsymbol{u}$ is the weak solution of the Navier-Stokes problem $(\mathcal{N S})$ given by Theorem 2.2. Then we rewrite the Navier-Stokes problem $(\mathcal{N S})$ as follows:

$$
(\mathcal{N S}) \begin{cases}-\nu \Delta \boldsymbol{v}+\lambda \frac{\partial \boldsymbol{v}}{\partial x_{1}}+\nabla \pi=\boldsymbol{f}-\boldsymbol{v} \cdot \nabla \boldsymbol{v} & \text { in } \mathbb{R}^{3} \\ \operatorname{div} \boldsymbol{v}=0 & \text { in } \mathbb{R}^{3} \\ \boldsymbol{v} \longrightarrow 0 & \text { if }|x| \rightarrow \infty\end{cases}
$$

Remark that the Navier-Stokes problem is reduced to the Oseen problem or the Stokes one, according to whether $\boldsymbol{u}_{\infty}$ is different from or equal to zero. However, if $\boldsymbol{u}_{\infty}=0$, several fundamental questions remain open. For instance, we cannot do when $\boldsymbol{u}_{\infty}=0$ is to show that $\boldsymbol{v} \in \mathbf{L}^{q}\left(\mathbb{R}^{3}\right)$ for some $q<6$ or $\nabla \boldsymbol{v} \in \mathbf{L}^{r}\left(\mathbb{R}^{3}\right)$ for some $r<2$ excepting the case where the forces are small in suitable norm (see Galdi [11], Farwig [10] for example). When $\boldsymbol{u}_{\infty} \neq 0$, the situation is different. Thanks to the results obtained on the Oseen system, we shall see here that the weak solutions satisfy the regularity properties according to $f$. We start our studies by adding assumptions on the force field $f$. First, we assume additionally that $\boldsymbol{f} \in \mathbf{W}_{0}^{-1,3}\left(\mathbb{R}^{3}\right)$, and then, we will consider the case more generally $\boldsymbol{f} \in \mathbf{W}_{0}^{-1,2}\left(\mathbb{R}^{3}\right) \cap \mathbf{W}_{0}^{-1, p}\left(\mathbb{R}^{3}\right)$ with $p \geq 3$. Following this idea, we state and prove the

Theorem 3.1. Given $p \geq 3$ and $\boldsymbol{f} \in \mathbf{W}_{0}^{-1,2}\left(\mathbb{R}^{3}\right) \cap \mathbf{W}_{0}^{-1, p}\left(\mathbb{R}^{3}\right)$. Then, each weak solution $\boldsymbol{u}$ to the problem $(\mathcal{N S})$ satisfies

$$
\boldsymbol{v} \in \mathbf{W}_{0}^{1,2}\left(\mathbb{R}^{3}\right) \cap \mathbf{W}_{0}^{1, p}\left(\mathbb{R}^{3}\right) \cap \mathbf{L}^{r_{1}}\left(\mathbb{R}^{3}\right) \text { and } \frac{\partial \boldsymbol{v}}{\partial x_{1}} \in \mathbf{W}_{0}^{-1, r_{2}}\left(\mathbb{R}^{3}\right)
$$

for any $r_{1} \geq 6$ and any $r_{2} \geq 3$. Besides, the associated pressure has a representative

$$
\pi \in L^{3}\left(\mathbb{R}^{3}\right) \cap L^{p}\left(\mathbb{R}^{3}\right),
$$

and if $p>3$, then we have $\boldsymbol{v} \in \mathbf{L}^{\infty}\left(\mathbb{R}^{3}\right)$.

Proof. We first prove the case $p=3$ and then consider the case $p>3$.

a) The case $p=3: \quad f \in \mathbf{W}_{0}^{-1,2}\left(\mathbb{R}^{3}\right) \cap \mathbf{W}_{0}^{-1,3}\left(\mathbb{R}^{3}\right)$. Let $\boldsymbol{u}$ be a weak solution of $(\mathcal{N S})$ given by Theorem 2.2 and $\boldsymbol{v}=\boldsymbol{u}-\boldsymbol{u}_{\infty}$. Since $\boldsymbol{v} \in \mathbf{L}^{6}\left(\mathbb{R}^{3}\right)$ and $\boldsymbol{v} . \nabla \boldsymbol{v}=\operatorname{div}(\boldsymbol{v} \otimes \boldsymbol{v})$, we have that $\boldsymbol{v} . \nabla \boldsymbol{v} \in \mathbf{W}_{0}^{-1,3}\left(\mathbb{R}^{3}\right)$ and $\boldsymbol{f}-\boldsymbol{v} . \nabla \boldsymbol{v} \in \mathbf{W}_{0}^{-1,3}\left(\mathbb{R}^{3}\right)$. Therefore, from Theorem 1.11, the following Oseen system

$$
-\nu \Delta \boldsymbol{w}+\lambda \frac{\partial \boldsymbol{w}}{\partial x_{1}}+\nabla q=\boldsymbol{f}-\boldsymbol{v} . \nabla \boldsymbol{v} \text { and } \operatorname{div} \boldsymbol{w}=0 \text { in } \mathbb{R}^{3}
$$


has a unique solution $(\boldsymbol{w}, q) \in\left(\widetilde{\mathbf{W}}_{0}^{1,3}\left(\mathbb{R}^{3}\right) \times L^{3}\left(\mathbb{R}^{3}\right)\right)$ such that $\boldsymbol{w} \in \mathbf{L}^{r}\left(\mathbb{R}^{3}\right)$ for any $r \geq 12$. We set $\boldsymbol{z}=\boldsymbol{v}-\boldsymbol{w}$ and $\theta=\pi-q$. Subtracting (3.1) to (3.4), we get

$$
-\nu \Delta \boldsymbol{z}+\lambda \frac{\partial \boldsymbol{z}}{\partial x_{1}}+\nabla \theta=\mathbf{0} \text { and } \operatorname{div} \boldsymbol{z}=0 \text { in } \mathbb{R}^{3} .
$$

Therefore, we have

$$
-\nu \Delta \operatorname{curl} \boldsymbol{z}+\lambda \frac{\partial(\operatorname{curl} \boldsymbol{z})}{\partial x_{1}}=\mathbf{0} \quad \text { in } \mathbb{R}^{3},
$$

and we get $\boldsymbol{\Psi}=\operatorname{curl} \boldsymbol{z}$, then for each $i=1,2,3$,

$$
-\nu \Delta \Psi_{i}+\lambda \frac{\partial \Psi_{i}}{\partial x_{1}}=0 \quad \text { in } \mathbb{R}^{3}
$$

where $\Psi_{i} \in L^{2}\left(\mathbb{R}^{3}\right)+L^{3}\left(\mathbb{R}^{3}\right) \hookrightarrow S^{\prime}\left(\mathbb{R}^{3}\right)$. Then, from Lemma $4.1[8], \Psi$ is a polynomial which belongs to $\mathbf{L}^{2}\left(\mathbb{R}^{3}\right)+\mathbf{L}^{3}\left(\mathbb{R}^{3}\right)$. Consequently, $\boldsymbol{\Psi}=\mathbf{0}=$ curl $\boldsymbol{z}$ and $\operatorname{div} \boldsymbol{z}=0$. Therefore,

$$
-\Delta \boldsymbol{z}=\operatorname{curl} \text { curl } \boldsymbol{z}+\nabla \operatorname{div} \boldsymbol{z}=\mathbf{0} \text { in } \mathbb{R}^{3} .
$$

Since $\boldsymbol{z}$ belongs to $\mathbf{W}_{0}^{1,2}\left(\mathbb{R}^{3}\right)+\mathbf{W}_{0}^{1,3}\left(\mathbb{R}^{3}\right)$, then $\boldsymbol{z}$ must be a constant $\boldsymbol{c}$ and $\nabla \boldsymbol{v}=\nabla \boldsymbol{w}$. As $\boldsymbol{z} \in \mathbf{L}^{6}\left(\mathbb{R}^{3}\right)+\mathbf{L}^{12}\left(\mathbb{R}^{3}\right)$, then $c=0$, i.e. $\boldsymbol{v}=\boldsymbol{w}$ and $\boldsymbol{v} \in$ $\mathbf{W}_{0}^{1,2}\left(\mathbb{R}^{3}\right) \cap \mathbf{W}_{0}^{1,3}\left(\mathbb{R}^{3}\right)$. Moreover, we have $\boldsymbol{v} \in \mathbf{L}^{r_{1}}\left(\mathbb{R}^{3}\right)$ and $\frac{\partial \boldsymbol{v}}{\partial x_{1}} \in \mathbf{W}_{0}^{-1, r_{2}}\left(\mathbb{R}^{3}\right)$ for any $r_{1} \geq 6$ and any $r_{2} \geq 3$. Since $\boldsymbol{z}=0$, we deduce that $\nabla \theta=0$, then $\theta$ must be a constant, i.e, $q=\pi+a$ with $a \in \mathbb{R}, q \in L^{3}\left(\mathbb{R}^{3}\right)$. This ends the proof of the case $p=3$.

b) The case $3<p<4$ : Let $f \in \mathbf{W}_{0}^{-1,2}\left(\mathbb{R}^{3}\right) \cap \mathbf{W}_{0}^{-1, p}\left(\mathbb{R}^{3}\right)$. It is clear that $\boldsymbol{f} \in \mathbf{W}_{0}^{-1,3}\left(\mathbb{R}^{3}\right)$ and since we have proved the theorem for $p=3$, we know that $\boldsymbol{v} \in \mathbf{W}_{0}^{1,2}\left(\mathbb{R}^{3}\right) \cap \mathbf{W}_{0}^{1,3}\left(\mathbb{R}^{3}\right) \cap \mathbf{L}^{r_{1}}\left(\mathbb{R}^{3}\right)$ for any $r_{1} \geq 6$, and $\pi \in L^{3}\left(\mathbb{R}^{3}\right)$. Since $\operatorname{div}(\boldsymbol{v} \otimes \boldsymbol{v}) \in \mathbf{W}_{0}^{-1, r}\left(\mathbb{R}^{3}\right)$ for all $r \geq 3$, by Theorem 1.11 , we can deduce as previously that $\boldsymbol{v} \in \mathbf{W}_{0}^{1,2}\left(\mathbb{R}^{3}\right) \cap \mathbf{W}_{0}^{1, p}\left(\mathbb{R}^{3}\right)$ with $\frac{\partial \boldsymbol{v}}{\partial x_{1}} \in \mathbf{W}_{0}^{-1, r_{2}}\left(\mathbb{R}^{3}\right)$ for all $r_{2} \geq 3$. Moreover, we can check that $\pi \in L^{3}\left(\mathbb{R}^{3}\right) \cap L^{p}\left(\mathbb{R}^{3}\right)$. This ends the proof of the case $3<p<4$.

c) The case $p \geq 4$ : From the case a) and b), we have $\boldsymbol{v} \in \mathbf{W}_{0}^{1, k}\left(\mathbb{R}^{3}\right), \boldsymbol{v} \in \mathbf{L}^{r_{1}}\left(\mathbb{R}^{3}\right)$, $\frac{\partial \boldsymbol{v}}{\partial x_{1}} \in \mathbf{W}_{0}^{-1, r_{2}}\left(\mathbb{R}^{3}\right)$ and $\pi \in L^{s}\left(\mathbb{R}^{3}\right)$ for all $q \in[2,4), k \in[2,4), r_{1} \in[6, \infty)$, $r_{2} \in[3, \infty)$ and $s \in[3,4)$. We use the same method of precedent cases, by applying Theorem 1.11, we can remark that $\nabla \boldsymbol{w}=\nabla \boldsymbol{v}$ even if $\boldsymbol{w} \in \mathbf{W}_{0}^{1, p}\left(\mathbb{R}^{3}\right)$ is unique up to an element of $\mathcal{N}_{0}$ and we still have (3.2) and (3.3).

If $p>3$, we have $\nabla \boldsymbol{v} \in \mathbf{L}^{p}\left(\mathbb{R}^{3}\right)$ and $\boldsymbol{v} \in \mathbf{L}^{r_{1}}\left(\mathbb{R}^{3}\right)$ for any $r_{1} \geq 6$. Hence, we deduce $\boldsymbol{v} \in \mathbf{L}^{\infty}\left(\mathbb{R}^{3}\right)$. The proof is complete.

From Sobolev injections theorem and the properties of the duality, we know that $\mathbf{L}^{3 / 2}\left(\mathbb{R}^{3}\right) \hookrightarrow \mathbf{W}_{0}^{-1,3}\left(\mathbb{R}^{3}\right)$. Then, if we reinforce the assumptions of Theorem $3.1, \boldsymbol{f}$ belongs to $\mathbf{L}^{3 / 2}\left(\mathbb{R}^{3}\right)$ instead of $\mathbf{W}_{0}^{-1,3}\left(\mathbb{R}^{3}\right)$, we can prove the following. 
Theorem 3.2. i) Assume that $\boldsymbol{f} \in \mathbf{W}_{0}^{-1,2}\left(\mathbb{R}^{3}\right) \cap \mathbf{L}^{3 / 2}\left(\mathbb{R}^{3}\right)$. Then each weak solution $\boldsymbol{u}$ to the problem $(\mathcal{N S})$ satisfies

$$
\begin{gathered}
\boldsymbol{v} \in \mathbf{W}_{0}^{1,2}\left(\mathbb{R}^{3}\right) \cap \mathbf{W}_{0}^{1,3}\left(\mathbb{R}^{3}\right) \cap \mathbf{L}^{r_{1}}\left(\mathbb{R}^{3}\right), \\
\frac{\partial \boldsymbol{v}}{\partial x_{1}} \in \mathbf{L}^{3 / 2}\left(\mathbb{R}^{3}\right) \cap \mathbf{L}^{3}\left(\mathbb{R}^{3}\right) \cap \mathbf{W}_{0}^{-1, r_{2}}\left(\mathbb{R}^{3}\right) \quad \text { and } \nabla^{2} \boldsymbol{v} \in \mathbf{L}^{3 / 2}\left(\mathbb{R}^{3}\right)
\end{gathered}
$$

for any $r_{1} \geq \frac{9}{2}, r_{2} \geq 3$. Besides, the associated pressure $\pi$ has a representative in $W_{0}^{1,3 / 2}\left(\mathbb{R}^{3}\right)$.

ii) Let $\frac{3}{2}<p<3$. Assume that $\boldsymbol{f} \in \mathbf{W}_{0}^{-1,2}\left(\mathbb{R}^{3}\right) \cap \mathbf{L}^{p}\left(\mathbb{R}^{3}\right)$. Then each solution $\boldsymbol{u}$ to the problem $(\mathcal{N S})$ satisfies

$$
\boldsymbol{v} \in \mathbf{W}_{0}^{1,2}\left(\mathbb{R}^{3}\right) \cap \mathbf{W}_{0}^{1, p *}\left(\mathbb{R}^{3}\right) \cap \mathbf{L}^{r_{1}}\left(\mathbb{R}^{3}\right) \text { and } \frac{\partial \boldsymbol{v}}{\partial x_{1}} \in \mathbf{W}_{0}^{-1, r_{2}}\left(\mathbb{R}^{3}\right)
$$

for any $r_{1} \in[3 p, \infty]$ if $\frac{3}{2}<p<2$, for any $r_{1} \in[6, \infty]$ if $2 \leq p<3$ and for any $r_{2} \geq 3$. Besides, the associated pressure has a representative

$$
\pi \in L^{3}\left(\mathbb{R}^{3}\right) \cap L^{p *}\left(\mathbb{R}^{3}\right)
$$

where $p *=\frac{3 p}{3-p}$. Moreover, we have

$$
\nabla^{2} \boldsymbol{v} \in \mathbf{L}^{p}\left(\mathbb{R}^{3}\right), \frac{\partial \boldsymbol{v}}{\partial x_{1}} \in \mathbf{L}^{p}\left(\mathbb{R}^{3}\right) \text { and } \pi \in W_{0}^{1, p}\left(\mathbb{R}^{3}\right) .
$$

Proof. i) Note that $\mathbf{L}^{3 / 2}\left(\mathbb{R}^{3}\right) \hookrightarrow \mathbf{W}_{0}^{-1,3}\left(\mathbb{R}^{3}\right)$ and let $\boldsymbol{u}$ be a weak solution of $(\mathcal{N S})$. Thanks to Theorem 3.1, we know that $\boldsymbol{u}$ and $\pi$ satisfy (3.2) and (3.3) for the case $p=3$. Besides, we have $\boldsymbol{f}-\boldsymbol{v} \cdot \nabla \boldsymbol{v}$ belongs to $\mathbf{L}^{3 / 2}\left(\mathbb{R}^{3}\right)$. Then, by applying Theorem 1.10, the following Oseen system

$$
-\nu \Delta \boldsymbol{w}+\lambda \frac{\partial \boldsymbol{w}}{\partial x_{1}}+\nabla \mu=\boldsymbol{f}-\boldsymbol{v} \cdot \nabla \boldsymbol{v} \text { and } \operatorname{div} \boldsymbol{w}=0 \text { in } \mathbb{R}^{3},
$$

has a solution $\boldsymbol{w} \in \mathbf{L}^{s}\left(\mathbb{R}^{3}\right)$ such that, $\nabla \boldsymbol{w} \in \mathbf{L}^{r}\left(\mathbb{R}^{3}\right), \nabla^{2} \boldsymbol{w} \in \mathbf{L}^{3 / 2}\left(\mathbb{R}^{3}\right), \frac{\partial \boldsymbol{w}}{\partial x_{1}} \in$ $\mathbf{L}^{3 / 2}\left(\mathbb{R}^{3}\right)$ and the pressure $\mu \in W_{0}^{1,3 / 2}\left(\mathbb{R}^{3}\right)$ for all $s \in[6, \infty)$ and $r \in[12 / 5,3]$. We set $\boldsymbol{z}=\boldsymbol{v}-\boldsymbol{w}$ and $\theta=\pi-\mu$. Subtracting (3.1) to (3.10), we get

$$
-\nu \Delta \boldsymbol{z}+\lambda \frac{\partial \boldsymbol{z}}{\partial x_{1}}+\nabla \theta=\mathbf{0} \text { and } \operatorname{div} \boldsymbol{z}=0 \text { in } \mathbb{R}^{3} .
$$

By the analogous techniques as in the proof of Theorem 3.1, we conclude $\boldsymbol{v}=\boldsymbol{w}$ and $\pi=\mu \in W_{0}^{1,3 / 2}\left(\mathbb{R}^{3}\right)$. Then, $\frac{\partial \boldsymbol{v}}{\partial x_{1}} \in \mathbf{L}^{3 / 2}\left(\mathbb{R}^{3}\right) \cap \mathbf{L}^{3}\left(\mathbb{R}^{3}\right)$ and $\nabla^{2} \boldsymbol{v} \in \mathbf{L}^{3 / 2}\left(\mathbb{R}^{3}\right)$. Thanks to Lemma 1.5 with $q=\frac{3}{2}$, we can deduce $\boldsymbol{v} \in \mathbf{L}^{9 / 2}\left(\mathbb{R}^{3}\right)$. Combining these results with (3.2) and (3.3), we obtain (3.5) and (3.6).

ii) Thanks to the Sobolev embedding theorem, since $\boldsymbol{f} \in \mathbf{L}^{p}\left(\mathbb{R}^{3}\right)$ where $\frac{3}{2}<$ $p<3$, we can deduce that $\boldsymbol{f} \in \mathbf{W}_{0}^{-1, p *}\left(\mathbb{R}^{3}\right)$ and $p *>3$. From Theorem 3.1, we 
have $\boldsymbol{v} \in \mathbf{W}_{0}^{1,2}\left(\mathbb{R}^{3}\right) \cap \mathbf{W}_{0}^{1, p *}\left(\mathbb{R}^{3}\right) \cap \mathbf{L}^{\infty}\left(\mathbb{R}^{3}\right)$. In particular, we have $\nabla \boldsymbol{v} \in \mathbf{L}^{q_{1}}\left(\mathbb{R}^{3}\right)$ for all $2 \leq q_{1} \leq 3$. Then, from Hölder's inequality, we obtain $\boldsymbol{v} \cdot \nabla \boldsymbol{v} \in \mathbf{L}^{q_{2}}\left(\mathbb{R}^{3}\right)$ for all $\frac{3}{2} \leq q_{2}<3$. Therefore, we deduce that $\boldsymbol{f}-\boldsymbol{v} . \nabla \boldsymbol{v} \in \mathbf{L}^{p}\left(\mathbb{R}^{3}\right)$. By using the methods as in the proof of Theorem 3.1 (part a) and from Theorem 1.10 for the case $\frac{3}{2} \leq p<2$, we have $\boldsymbol{v} \in \mathbf{L}^{s}\left(\mathbb{R}^{3}\right)$ where $s \geq \frac{2 p}{2-p}$, $\pi \in W_{0}^{1, p}\left(\mathbb{R}^{3}\right), \nabla \boldsymbol{v} \in \mathbf{L}^{r}\left(\mathbb{R}^{3}\right)$ where $\frac{4 p}{4-p} \leq r \leq \frac{3 p}{3-p}, \nabla^{2} \boldsymbol{v} \in \mathbf{L}^{p}\left(\mathbb{R}^{3}\right)$ and $\frac{\partial \boldsymbol{v}}{\partial x_{1}} \in \mathbf{L}^{p}\left(\mathbb{R}^{3}\right)$. We note that $6 \leq \frac{2 p}{2-p}$ and $4 \leq \frac{4 p}{4-p}<6 \leq \frac{3 p}{3-p}$. Since $\boldsymbol{v} \in \mathbf{W}_{0}^{1,2}\left(\mathbb{R}^{3}\right) \cap \mathbf{W}_{0}^{1, p *}\left(\mathbb{R}^{3}\right) \cap \mathbf{L}^{r_{1}}\left(\mathbb{R}^{3}\right)$ for any $r_{1} \in[6, \infty]$, we don't obtain more results for $\boldsymbol{v}$. But by applying Lemma 1.5, we have $\boldsymbol{v} \in \mathbf{L}^{3 p}\left(\mathbb{R}^{3}\right)$. Proceeding analogously for the case $2 \leq p<3$, we have $\boldsymbol{v} \in \mathbf{W}_{0}^{1, r}\left(\mathbb{R}^{3}\right)$ where $\frac{4 p}{4-p} \leq r \leq \frac{3 p}{3-p}, \pi \in W_{0}^{1, p}\left(\mathbb{R}^{3}\right), \nabla^{2} \boldsymbol{v} \in \mathbf{L}^{p}\left(\mathbb{R}^{3}\right)$ and $\frac{\partial \boldsymbol{v}}{\partial x_{1}} \in \mathbf{L}^{p}\left(\mathbb{R}^{3}\right)$. Remark that $2<\frac{4 p}{4-p}<\frac{3 p}{3-p}=p *$, then $\boldsymbol{v}$ and $\pi$ can not be improved on and we shall keep all results in (3.7), (3.8) and (3.9) for any $r_{1} \in[6, \infty]$ and $r_{2} \geq 3$. The theorem is completely proved.

\section{More regularity}

For our studies, we shall introduce the following problem. Let a fixed $\boldsymbol{z} \in \mathbf{L}^{3}\left(\mathbb{R}^{3}\right)$ such that $\operatorname{div} \boldsymbol{z}=0$ in $\mathbb{R}^{3}$, we search a solution $(\boldsymbol{w}, \theta)$ to the following problem

$$
\begin{aligned}
-\nu \Delta \boldsymbol{w}+\lambda \frac{\partial \boldsymbol{w}}{\partial x_{1}}+\boldsymbol{z} \cdot \nabla \boldsymbol{w}+\nabla \theta=\boldsymbol{f} & \text { in } \mathbb{R}^{3}, \\
\operatorname{div} \boldsymbol{w}=0 & \text { in } \mathbb{R}^{3} .
\end{aligned}
$$

This problem is here linear, we limit ourselves to the condition $\boldsymbol{w} \rightarrow \mathbf{0}$ at infinity. This condition is satisfied if $p<3$ and $\boldsymbol{w} \in \mathbf{W}_{0}^{1, p}\left(\mathbb{R}^{3}\right)$ or $\boldsymbol{w} \in \mathbf{L}^{q}\left(\mathbb{R}^{3}\right)$ for some $q \geq 1$ and $\boldsymbol{w} \in \mathbf{W}_{0}^{1, p}\left(\mathbb{R}^{3}\right)$ if $p \geq 3$ (see [7]).

We now prove the

Lemma 4.1. Assume that $\boldsymbol{z} \in \mathbf{L}^{3}\left(\mathbb{R}^{3}\right)$ with $\operatorname{div} \boldsymbol{z}=0$ and let $\boldsymbol{f} \in \mathbf{W}_{0}^{-1,2}\left(\mathbb{R}^{3}\right)$. Then Problem (4.1) has a unique solution $(\boldsymbol{w}, \theta) \in \mathbf{W}_{0}^{1,2}\left(\mathbb{R}^{3}\right) \times L^{2}\left(\mathbb{R}^{3}\right)$. Moreover, we have $\boldsymbol{w} \in \mathbf{L}^{4}\left(\mathbb{R}^{3}\right), \frac{\partial \boldsymbol{w}}{\partial x_{1}} \in \mathbf{W}_{0}^{-1,2}\left(\mathbb{R}^{3}\right)$ and $\boldsymbol{w}$ satisfies the energy equality

$$
\nu \int_{\mathbb{R}^{3}}|\nabla \boldsymbol{w}|^{2} d \boldsymbol{x}=<\boldsymbol{f}, \boldsymbol{w}>_{\mathbf{W}_{0}^{-1,2}\left(\mathbb{R}^{3}\right) \times \mathbf{W}_{0}^{1,2}\left(\mathbb{R}^{3}\right)} .
$$

Proof. Let $\left(R_{m}\right)_{m \geq 0}$ be an increasing sequence of reals with a fixed $R_{0}>0$ and such that $\lim _{m \rightarrow+\infty} R_{m}=+\infty$. Since $f \in \mathbf{W}_{0}^{-1,2}\left(\mathbb{R}^{3}\right)$, then its restriction to the open ball of radius $R_{m}>0$ belongs to $\mathbf{H}^{-1}\left(B_{m}\right)$. Now proceeding as in Theorem 2.2 , we can deduce that the following approximating problem

$$
\begin{aligned}
-\nu \Delta \boldsymbol{w}_{m}+\lambda \frac{\partial \boldsymbol{w}_{m}}{\partial x_{1}}+\boldsymbol{z} \cdot \nabla \boldsymbol{w}_{m}+\nabla \theta_{m}=\boldsymbol{f} & \text { in } B_{m}, \\
\operatorname{div} \boldsymbol{w}_{m}=0 & \text { in } B_{m}, \\
\boldsymbol{w}_{m}=0 & \text { on } \partial B_{m} .
\end{aligned}
$$


has a unique solution $\left(\boldsymbol{w}_{m}, \theta_{m}\right) \in \mathbf{H}^{1}\left(B_{m}\right) \times\left(L^{2}\left(B_{m}\right) / \mathbb{R}\right)$. Extending $\boldsymbol{w}_{m}$ and $\theta_{m}$ by zero outside $B_{m}$ and when $m \rightarrow+\infty$, we can prove analogously as in Theorem 2.2 that Problem (4.1) has a weak solution $(\boldsymbol{w}, \chi) \in \mathbf{W}_{0}^{1,2}\left(\mathbb{R}^{3}\right) \times L_{\text {loc }}^{2}\left(\mathbb{R}^{3}\right)$. It is easy to check that $\Delta \boldsymbol{w}$ and $\boldsymbol{z} \cdot \nabla \boldsymbol{w}=\operatorname{div}(\boldsymbol{z} \otimes \boldsymbol{w})$ belong to $\mathbf{W}_{0}^{-1,2}\left(\mathbb{R}^{3}\right)$. Then from (4.1), we have $\nabla \chi \in \mathbf{L}^{2}\left(\mathbb{R}^{3}\right)+\mathbf{W}_{0}^{-1,2}\left(\mathbb{R}^{3}\right)$. In addition, since $\boldsymbol{z} \otimes \boldsymbol{w} \in$ $\mathbf{L}^{2}\left(\mathbb{R}^{3}\right)$, we have

$$
\Delta \chi=\operatorname{div} \boldsymbol{f}-\operatorname{div} \operatorname{div}(\boldsymbol{z} \otimes \boldsymbol{w}) .
$$

The right-hand side of (4.4) being a element of $\mathbf{W}_{0}^{-2,2}\left(\mathbb{R}^{3}\right) \perp \mathbb{R}$, then there exists a unique $\theta \in L^{2}\left(\mathbb{R}^{3}\right)$ such that $\Delta \theta=\Delta \chi$. Thus, $\nabla(\theta-\chi)$ is a harmonic distribution belonging to $\mathbf{W}_{0}^{-1,2}\left(\mathbb{R}^{3}\right)+\mathbf{L}^{2}\left(\mathbb{R}^{3}\right)$, i.e, $\nabla \chi=\nabla \theta$. Then, there exists $k \in \mathbb{R}$ such that $\theta=\chi+k \in L^{2}\left(\mathbb{R}^{3}\right)$. Moreover, we have $\frac{\partial \boldsymbol{w}}{\partial x_{1}} \in \mathbf{W}_{0}^{-1,2}\left(\mathbb{R}^{3}\right)$ because $\Delta \boldsymbol{w}, \boldsymbol{z} \cdot \nabla \boldsymbol{w}, \nabla \theta$ and $\boldsymbol{f}$ belong to $\mathbf{W}_{0}^{-1,2}\left(\mathbb{R}^{3}\right)$. Thanks to Lemma 1.6, we deduce $\boldsymbol{w} \in \mathbf{L}^{4}\left(\mathbb{R}^{3}\right)$. It is easy to check as in the proof of Lemma 1.6 that

$$
\left\langle\frac{\partial \boldsymbol{w}}{\partial x_{1}}, \boldsymbol{w}\right\rangle=\langle\nabla \theta, \boldsymbol{w}\rangle=\langle\operatorname{div}(\boldsymbol{z} \otimes \boldsymbol{w}), \boldsymbol{w}\rangle=0,
$$

where the brackets denote the duality $\mathbf{W}_{0}^{-1,2}\left(\mathbb{R}^{3}\right) \times \mathbf{W}_{0}^{1,2}\left(\mathbb{R}^{3}\right)$. Therefore, we obtain the energy equality (4.2).

We now introduce the following results which we shall need in the future.

Lemma 4.2. Let $\boldsymbol{z} \in \mathbf{L}^{4}\left(\mathbb{R}^{3}\right)$ such that $\operatorname{div} \boldsymbol{z}=0$. Then, for all $\varepsilon>0$, there exist $\rho=\rho(\varepsilon, \boldsymbol{z})>0$ and a sequence $\left(\boldsymbol{z}_{k}\right)_{k \in \mathbb{N}} \in \mathbf{L}^{3}\left(\mathbb{R}^{3}\right) \cap \mathbf{L}^{4}\left(\mathbb{R}^{3}\right)$, such that $\operatorname{div} \boldsymbol{z}_{k}=0$, satisfying

$$
\boldsymbol{z}_{k} \rightarrow \boldsymbol{z} \text { in } \mathbf{L}^{4}\left(\mathbb{R}^{3}\right) \text {. }
$$

Moreover, there exist sequences $\left(\boldsymbol{a}_{k}\right)$ and $\left(\boldsymbol{b}_{k}\right)$ in $\mathbf{L}^{3}\left(\mathbb{R}^{3}\right) \cap \mathbf{L}^{4}\left(\mathbb{R}^{3}\right)$ satisfying for each $k \in \mathbb{N}$

$$
\boldsymbol{z}_{k}=\boldsymbol{a}_{k}+\boldsymbol{b}_{k} \text { with }\left\|\boldsymbol{a}_{k}\right\|_{\mathbf{L}^{4}\left(\mathbb{R}^{3}\right)} \leq \varepsilon \text { and } \operatorname{supp} \boldsymbol{b}_{k} \subset B(0, \rho) .
$$

Proof. Let $\varphi \in C^{\infty}\left(\mathbb{R}^{+}\right)$such that $0 \leq \varphi \leq 1$ satisfying $\varphi(t)=1$ if $0 \leq t \leq 1$ and $\varphi(t)=0$ if $t \geq 2$. For $a>0$, we set

$$
\varphi_{a}(x)=\varphi\left(\frac{|x|}{a}\right), \quad x \in \mathbb{R}^{3} .
$$

Let $\varepsilon>0$, then there exists $\rho=\rho(\varepsilon, \boldsymbol{z})>0$ such that

$$
\left\|\boldsymbol{z}-\varphi_{\rho} \boldsymbol{z}\right\|_{\mathbf{L}^{4}\left(\mathbb{R}^{3}\right)} \leq \frac{\varepsilon}{2} .
$$

Let $\left(R_{k}\right)_{k \in \mathbb{N}}$ be an increasing unbounded sequence of positive numbers with $R_{0}>2 \rho$. Since the support of $\varphi_{R_{k}}$ is compact for all $k \in \mathbb{N}$, then $\operatorname{div}\left(\varphi_{R_{k}} z\right)=$ $\boldsymbol{z} . \nabla \varphi_{R_{k}}$ belongs to $\mathbf{L}^{4}\left(\mathbb{R}^{3}\right)$ and has a compact support. In particular, div $\left(\varphi_{R_{k}} \boldsymbol{z}\right)$ belongs to $\mathbf{L}^{3 / 2}\left(\mathbb{R}^{3}\right) \cap \mathbf{L}^{12 / 7}\left(\mathbb{R}^{3}\right)$, and from [5], we deduce that there exists $\boldsymbol{y}_{k}$ $\in \mathbf{W}_{0}^{1,3 / 2}\left(\mathbb{R}^{3}\right) \cap \mathbf{W}_{0}^{1,12 / 7}\left(\mathbb{R}^{3}\right)$ such that $\operatorname{div} \boldsymbol{y}_{k}=-\operatorname{div}\left(\varphi_{R_{k}} \boldsymbol{z}\right)$ satisfying the following estimation

$$
\begin{aligned}
\left\|\boldsymbol{y}_{k}\right\|_{\mathbf{L}^{4}\left(\mathbb{R}^{3}\right)} & \leq C\left\|\boldsymbol{z} \cdot \nabla \varphi_{R_{k}}\right\|_{\mathbf{L}^{12 / 7}\left(\mathbb{R}^{3}\right)} \\
& \leq C\|\boldsymbol{z}\|_{\mathbf{L}^{4}\left(B_{R_{k}}^{2 R_{k}}\right)}\left\|\nabla \varphi_{R_{k}}\right\|_{\mathbf{L}^{3}\left(B_{R_{k}}^{2 R_{k}}\right)} \\
& \leq C\|\boldsymbol{z}\|_{\mathbf{L}^{4}\left(B_{R_{k}}^{2 R_{k}}\right)} .
\end{aligned}
$$


Here, $B_{R_{k}}$ is a open ball of radius $R_{k}>0$ centered at the origin and $B_{R_{k}}^{2 R_{k}}=$ $B_{2 R_{k}} \backslash B_{R_{k}}$. Note that $\mathbf{W}_{0}^{1,12 / 7}\left(\mathbb{R}^{3}\right) \hookrightarrow \mathbf{L}^{4}\left(\mathbb{R}^{3}\right)$ and $\mathbf{W}_{0}^{1,3 / 2}\left(\mathbb{R}^{3}\right) \hookrightarrow \mathbf{L}^{3}\left(\mathbb{R}^{3}\right)$. We define $\boldsymbol{z}_{k}=\varphi_{R_{k}} \boldsymbol{z}+\boldsymbol{y}_{k}$. Then, from (4.8), we have (4.6). We set that

$$
\boldsymbol{z}_{k}=\varphi_{R_{k}} \boldsymbol{z}+\boldsymbol{y}_{k}=\left[\varphi_{R_{k}}\left(1-\varphi_{\rho}\right) \boldsymbol{z}+\boldsymbol{y}_{k}\right]+\left(\varphi_{R_{k}} \varphi_{\rho} \boldsymbol{z}\right)=: \boldsymbol{a}_{k}+\boldsymbol{b}_{k} .
$$

Note that supp $\boldsymbol{b}_{k} \subset B(0, \rho)$ and $\boldsymbol{b}_{k} \in \mathbf{L}^{3}\left(\mathbb{R}^{3}\right)$. Furthermore, for all $k \geq \bar{k}(\varepsilon) \in$ $\mathbb{N}$, we have

$$
\begin{aligned}
\left\|\boldsymbol{a}_{k}\right\|_{\mathbf{L}^{4}\left(\mathbb{R}^{3}\right)} & \leq\left\|\varphi_{R_{k}}\left(1-\varphi_{\rho}\right) \boldsymbol{z}\right\|_{\mathbf{L}^{4}\left(\mathbb{R}^{3}\right)}+\left\|\boldsymbol{y}_{k}\right\|_{\mathbf{L}^{4}\left(\mathbb{R}^{3}\right)} \\
& \leq \frac{\varepsilon}{2}+C\|\boldsymbol{z}\|_{\mathbf{L}^{4}\left(B_{R_{k}}^{2 R_{k}}\right)} \leq \frac{\varepsilon}{2}+\frac{\varepsilon}{2}=\varepsilon
\end{aligned}
$$

and we obtain (4.7). Moreover, since $\boldsymbol{y}_{k} \in \mathbf{L}^{3}\left(\mathbb{R}^{3}\right) \cap \mathbf{L}^{4}\left(\mathbb{R}^{3}\right)$, we have also $\boldsymbol{a}_{k} \in \mathbf{L}^{3}\left(\mathbb{R}^{3}\right) \cap \mathbf{L}^{4}\left(\mathbb{R}^{3}\right)$.

In Theorem 3.2 (i), we proved $\boldsymbol{v} \in \mathbf{L}^{r_{1}}\left(\mathbb{R}^{3}\right)$ for any $r_{1} \geq 9 / 2$. To obtain $\boldsymbol{v} \in \mathbf{L}^{r_{1}}\left(\mathbb{R}^{3}\right)$ with $r_{1}<9 / 2$, we have to assume additionally a condition for $\boldsymbol{f}$. We can state the

Theorem 4.3. Assume that $\boldsymbol{f} \in \mathbf{W}_{0}^{-1,2}\left(\mathbb{R}^{3}\right) \cap \mathbf{L}^{3 / 2}\left(\mathbb{R}^{3}\right) \cap \mathbf{L}^{4 / 3}\left(\mathbb{R}^{3}\right)$. Then each weak solution $\boldsymbol{u}$ and the associate pressure $\pi$ to the problem $(\mathcal{N S})$ satisfy the results in Theorem 3.2 i). Moreover, for any $r_{1} \geq 4$

$$
\boldsymbol{v} \in \mathbf{L}^{r_{1}}\left(\mathbb{R}^{3}\right), \nabla^{2} \boldsymbol{v} \in \mathbf{L}^{4 / 3}\left(\mathbb{R}^{3}\right), \frac{\partial \boldsymbol{v}}{\partial x_{1}} \in \mathbf{L}^{4 / 3}\left(\mathbb{R}^{3}\right) \text { and } \pi \in W_{0}^{1,4 / 3}\left(\mathbb{R}^{3}\right) .
$$

Proof. From the case $i$ ) of Theorem 3.2, since $\boldsymbol{v} \in \mathbf{L}^{r_{1}}\left(\mathbb{R}^{3}\right)$ for any $r_{1} \geq 9 / 2$ and $\nabla \boldsymbol{v} \in \mathbf{L}^{2}\left(\mathbb{R}^{3}\right) \cap \mathbf{L}^{3}\left(\mathbb{R}^{3}\right)$, then we have $\boldsymbol{f}-\boldsymbol{v} . \nabla \boldsymbol{v} \in \mathbf{L}^{p}\left(\mathbb{R}^{3}\right)$ for any $p \in[18 / 13,3 / 2]$. From Theorem 1.10 and proceeding as in the proof of Theorem 3.1 with $p=$ $\frac{18}{13}$, we obtain $\frac{\partial \boldsymbol{v}}{\partial x_{1}} \in \mathbf{L}^{18 / 13}\left(\mathbb{R}^{3}\right), \nabla^{2} \boldsymbol{v} \in \mathbf{L}^{18 / 13}\left(\mathbb{R}^{3}\right)$ and $\pi \in W_{0}^{1,18 / 13}\left(\mathbb{R}^{3}\right)$. Moreover, we have

$$
\begin{aligned}
\lambda\left\|\frac{\partial \boldsymbol{v}}{\partial x_{1}}\right\|_{\mathbf{L}^{18 / 13}\left(\mathbb{R}^{3}\right)} & \leq C\|\boldsymbol{f}-\boldsymbol{v} \cdot \nabla \boldsymbol{v}\|_{\mathbf{L}^{18 / 13}\left(\mathbb{R}^{3}\right)} \\
& \leq C\left(\|\boldsymbol{f}\|_{\mathbf{L}^{18 / 13}\left(\mathbb{R}^{3}\right)}+\|\boldsymbol{v}\|_{\mathbf{L}^{9 / 2}\left(\mathbb{R}^{3}\right)}\|\nabla \boldsymbol{v}\|_{\mathbf{L}^{2}\left(\mathbb{R}^{3}\right)}\right) \\
& \leq C\left(\|\boldsymbol{f}\|_{\mathbf{L}^{18 / 13}\left(\mathbb{R}^{3}\right)}+\|\boldsymbol{v}\|_{\mathbf{L}^{9 / 2}\left(\mathbb{R}^{3}\right)}\|\boldsymbol{f}\|_{\mathbf{W}_{0}^{-1,2}\left(\mathbb{R}^{3}\right)}\right) .
\end{aligned}
$$

Applying Lemma 1.5, we have $\boldsymbol{v} \in \mathbf{L}^{54 / 13}\left(\mathbb{R}^{3}\right)$ and

$$
\|\boldsymbol{v}\|_{L^{54 / 13}\left(\mathbb{R}^{3}\right)} \leq C\left(\left\|\frac{\partial \boldsymbol{v}}{\partial x_{1}}\right\|_{\mathbf{L}^{18 / 13}\left(\mathbb{R}^{3}\right)}+\|\nabla \boldsymbol{v}\|_{\mathbf{L}^{2}\left(\mathbb{R}^{3}\right)}\right) .
$$

From (4.9) and (4.10), we deduce that

$$
\|\boldsymbol{v}\|_{L^{54 / 13\left(\mathbb{R}^{3}\right)}}+\lambda\left\|\frac{\partial \boldsymbol{v}}{\partial x_{1}}\right\|_{\mathbf{L}^{18 / 13}\left(\mathbb{R}^{3}\right)} \leq C\left(\|\boldsymbol{f}\|_{\mathbf{L}^{18 / 13}\left(\mathbb{R}^{3}\right)}+\|\boldsymbol{v}\|_{\mathbf{L}^{9 / 2}\left(\mathbb{R}^{3}\right)}+1\right) .
$$

Therefore, repeating the reasoning previously employed, we deduce for $1<q<$ $18 / 13$ that

$$
\|\boldsymbol{v}\|_{L^{3 q}\left(\mathbb{R}^{3}\right)}+\lambda\left\|\frac{\partial \boldsymbol{v}}{\partial x_{1}}\right\|_{\mathbf{L}^{q}\left(\mathbb{R}^{3}\right)} \leq C\left(\|\boldsymbol{f}\|_{\mathbf{L}^{q}\left(\mathbb{R}^{3}\right)}+\|\boldsymbol{v}\|_{\mathbf{L}^{2 q /(2-q)}\left(\mathbb{R}^{3}\right)}+1\right) .
$$


We define the sequence $\left\{q_{k}\right\}$ as follows

$$
\frac{2 q_{k+1}}{2-q_{k+1}}=3 q_{k}, \quad k \in \mathbb{N}
$$

with $q_{0}=18 / 13$. Repeating the same techniques, we thus find, for any $k \in \mathbb{N}$,

$$
\|\boldsymbol{v}\|_{L^{3 q_{k}\left(\mathbb{R}^{3}\right)}}+\left\|\frac{\partial \boldsymbol{v}}{\partial x_{1}}\right\|_{\mathbf{L}^{q_{k}\left(\mathbb{R}^{3}\right)}} \leq M
$$

for a constant $M$ independent of $k$. Clearly, the sequence $\left\{q_{k}\right\}$ is strictly decreasing and is bounded from below by $4 / 3$. Therefore, there exists a number $Q \geq 4 / 3$ such that

$$
\lim _{k \rightarrow \infty} q_{k}=Q .
$$

We shall pass to limit in (4.11), we obtain $Q=4 / 3$. Since $\boldsymbol{v} \in \mathbf{L}^{4}\left(\mathbb{R}^{3}\right)$ and $\nabla \boldsymbol{v} \in \mathbf{L}^{2}\left(\mathbb{R}^{3}\right)$, we obtain $\boldsymbol{f}-\boldsymbol{v} . \nabla \boldsymbol{v} \in \mathbf{L}^{4 / 3}\left(\mathbb{R}^{3}\right)$. Hence, by applying Theorem 1.10 , we can deduce that $\nabla^{2} \boldsymbol{v} \in \mathbf{L}^{4 / 3}\left(\mathbb{R}^{3}\right)$ and $\pi \in W_{0}^{1,4 / 3}\left(\mathbb{R}^{3}\right)$. The Theorem is completely proved.

Note that $\mathbf{L}^{6 / 5}\left(\mathbb{R}^{3}\right) \hookrightarrow \mathbf{W}_{0}^{-1,2}\left(\mathbb{R}^{3}\right)$ and $\mathbf{L}^{3 / 2}\left(\mathbb{R}^{3}\right) \hookrightarrow \mathbf{W}_{0}^{-1,3}\left(\mathbb{R}^{3}\right)$, and with the previous results in hand, we can now prove the following theorem.

Theorem 4.4. Let $\boldsymbol{f} \in \mathbf{L}^{6 / 5}\left(\mathbb{R}^{3}\right) \cap \mathbf{L}^{3 / 2}\left(\mathbb{R}^{3}\right)$. Then each weak solution $(\boldsymbol{u}, \pi)$ to the problem $(\mathcal{N S})$, satisfies

$$
\begin{gathered}
\boldsymbol{v} \in \mathbf{L}^{q}\left(\mathbb{R}^{3}\right) \text { for all } q \in[3, \infty), \pi \in W_{0}^{1,6 / 5}\left(\mathbb{R}^{3}\right) \cap W_{0}^{1,3 / 2}\left(\mathbb{R}^{3}\right), \\
\nabla \boldsymbol{v} \in \mathbf{L}^{12 / 7}\left(\mathbb{R}^{3}\right) \cap \mathbf{L}^{3}\left(\mathbb{R}^{3}\right), \nabla^{2} \boldsymbol{v} \in \mathbf{L}^{6 / 5}\left(\mathbb{R}^{3}\right) \cap \mathbf{L}^{3 / 2}\left(\mathbb{R}^{3}\right), \\
\frac{\partial \boldsymbol{v}}{\partial x_{1}} \in \mathbf{L}^{6 / 5}\left(\mathbb{R}^{3}\right) \cap \mathbf{L}^{3}\left(\mathbb{R}^{3}\right) .
\end{gathered}
$$

Proof. Let $\boldsymbol{u}$ be a weak solution of $(\mathcal{N S})$. As $f$ satisfies the hypothesis of Theorem 4.3, then $\boldsymbol{v} \in \mathbf{L}^{4}\left(\mathbb{R}^{3}\right)$ and $\frac{\partial \boldsymbol{v}}{\partial x_{1}} \in \mathbf{L}^{4 / 3}\left(\mathbb{R}^{3}\right)$. Let $\varepsilon>0, \rho>0$ and $\boldsymbol{v}_{k}$ be a sequence as $\boldsymbol{z}_{k}$ in Lemma 4.2. Since $\boldsymbol{v}_{k} \in \mathbf{L}^{3}\left(\mathbb{R}^{3}\right)$ and $\operatorname{div} \boldsymbol{v}_{k}=0$, from Lemma 4.1 , there exists a unique solution $\left(\boldsymbol{w}_{k}, \theta_{k}\right) \in \widetilde{\mathbf{W}}_{0}^{1,2}\left(\mathbb{R}^{3}\right) \times L^{2}\left(\mathbb{R}^{3}\right)$ satisfying

$$
-\nu \Delta \boldsymbol{w}_{k}+\lambda \frac{\partial \boldsymbol{w}_{k}}{\partial x_{1}}+\boldsymbol{v}_{k} . \nabla \boldsymbol{w}_{k}+\nabla \theta_{k}=\boldsymbol{f} \text { and } \operatorname{div} \boldsymbol{w}_{k}=0 \text { in } \mathbb{R}^{3} .
$$

Since $\boldsymbol{f}-\boldsymbol{v}_{k} . \nabla \boldsymbol{w}_{k} \in \mathbf{L}^{6 / 5}\left(\mathbb{R}^{3}\right)$, thanks to Theorem 1.10 , there exists a unique $\left(\boldsymbol{y}_{k}, \mu_{k}\right)$ such that

$$
-\nu \Delta \boldsymbol{y}_{k}+\lambda \frac{\partial \boldsymbol{y}_{k}}{\partial x_{1}}+\nabla \mu_{k}=\boldsymbol{f}-\boldsymbol{v}_{k} . \nabla \boldsymbol{w}_{k} \quad \text { and } \quad \operatorname{div} \boldsymbol{y}_{k}=0 \text { in } \mathbb{R}^{3},
$$

satisfying $\nabla^{2} \boldsymbol{y}_{k} \in \mathbf{L}^{6 / 5}\left(\mathbb{R}^{3}\right), \nabla \boldsymbol{y}_{k} \in \mathbf{L}^{12 / 7}\left(\mathbb{R}^{3}\right) \cap \mathbf{L}^{2}\left(\mathbb{R}^{3}\right), \boldsymbol{y}_{k} \in \mathbf{L}^{3}\left(\mathbb{R}^{3}\right) \cap \mathbf{L}^{6}\left(\mathbb{R}^{3}\right)$, $\frac{\partial \boldsymbol{y}_{k}}{\partial x_{1}} \in \mathbf{L}^{6 / 5}\left(\mathbb{R}^{3}\right)$ and $\mu_{k} \in W_{0}^{1,6 / 5}\left(\mathbb{R}^{3}\right)$. Using the method in the proof of Theorem 3.1 (part a), we have $\boldsymbol{y}_{k}=\boldsymbol{w}_{k}$ and $\mu_{k}=\theta_{k}$. Moreover, we have

$$
\begin{aligned}
& (\lambda \nu)^{1 / 2}\left\|\boldsymbol{w}_{k}\right\|_{\mathbf{L}^{3}\left(\mathbb{R}^{3}\right)}+\lambda^{1 / 4} \nu^{3 / 4}\left\|\nabla \boldsymbol{w}_{k}\right\|_{\mathbf{L}^{12 / 7}\left(\mathbb{R}^{3}\right)} \\
& +\lambda\left\|\frac{\partial \boldsymbol{w}_{k}}{\partial x_{1}}\right\|_{\mathbf{L}^{6 / 5}\left(\mathbb{R}^{3}\right)}+\nu\left\|\nabla^{2} \boldsymbol{w}_{k}\right\|_{\mathbf{L}^{6 / 5}\left(\mathbb{R}^{3}\right)}+\left\|\theta_{k}\right\|_{W_{0}^{1,6 / 5}\left(\mathbb{R}^{3}\right)} \\
& \leq C\left(\|\boldsymbol{f}\|_{\mathbf{L}^{6 / 5\left(\mathbb{R}^{3}\right)}}+\left\|\boldsymbol{v}_{k} . \nabla \boldsymbol{w}_{k}\right\|_{\mathbf{L}^{6 / 5}\left(\mathbb{R}^{3}\right)}\right) .
\end{aligned}
$$


Note now that

$$
\begin{aligned}
& \left\|\boldsymbol{v}_{k} . \nabla \boldsymbol{w}_{k}\right\|_{\mathbf{L}^{6 / 5}\left(\mathbb{R}^{3}\right)} \\
& \leq\left\|\boldsymbol{a}_{k}\right\|_{\mathbf{L}^{4}\left(\mathbb{R}^{3}\right)}\left\|\nabla \boldsymbol{w}_{k}\right\|_{\mathbf{L}^{12 / 7}\left(\mathbb{R}^{3}\right)}+\left\|\boldsymbol{b}_{k}\right\|_{\mathbf{L}^{6}\left(B_{\rho}\right)}\left\|\nabla \boldsymbol{w}_{k}\right\|_{\mathbf{L}^{3 / 2}\left(B_{\rho}\right)} \\
& \leq \varepsilon\left\|\nabla \boldsymbol{w}_{k}\right\|_{\mathbf{L}^{12 / 7}\left(\mathbb{R}^{3}\right)}+\|\boldsymbol{v}\|_{\mathbf{L}^{6}\left(\mathbb{R}^{3}\right)}\left\|\nabla \boldsymbol{w}_{k}\right\|_{\mathbf{L}^{3 / 2}\left(B_{\rho}\right)} .
\end{aligned}
$$

But there exists $C_{1} \in \mathbb{R}$ such that

$$
\forall k \in \mathbb{N}^{*},\left\|\nabla \boldsymbol{w}_{k}\right\|_{\mathbf{L}^{3 / 2}\left(B_{\rho}\right)} \leq C_{1}\|\boldsymbol{f}\|_{\mathbf{L}^{6 / 5}\left(\mathbb{R}^{3}\right)} .
$$

Contradicting (4.17) means that there exists a sequence $\left(k_{m}\right)_{m \in \mathbb{N}^{*}}$ such that, for all $m \in \mathbb{N}^{*}$,

$$
\begin{gathered}
\left\|\nabla \boldsymbol{w}_{k_{m}}\right\|_{\mathbf{L}^{3 / 2}\left(B_{\rho}\right)}=1, \\
\left\|-\nu \Delta \boldsymbol{w}_{k_{m}}+\lambda \frac{\partial \boldsymbol{w}_{k_{m}}}{\partial x_{1}}+\boldsymbol{v}_{k_{m}} . \nabla \boldsymbol{w}_{k_{m}}+\nabla \theta_{k_{m}}\right\|_{\mathbf{L}^{6 / 5}\left(\mathbb{R}^{3}\right)} \leq \frac{1}{m} .
\end{gathered}
$$

Then we deduce from (4.15), (4.16) and (4.18) that

$$
\begin{aligned}
& (\lambda \nu)^{1 / 2}\left\|\boldsymbol{w}_{k_{m}}\right\|_{\mathbf{L}^{3}\left(\mathbb{R}^{3}\right)}+\lambda^{1 / 4} \nu^{3 / 4}\left\|\nabla \boldsymbol{w}_{k_{m}}\right\|_{\mathbf{L}^{12 / 7}\left(\mathbb{R}^{3}\right)}+\nu\left\|\nabla^{2} \boldsymbol{w}_{k_{m}}\right\|_{\mathbf{L}^{6 / 5}\left(\mathbb{R}^{3}\right)} \\
& +\lambda\left\|\frac{\partial \boldsymbol{w}_{k_{m}}}{\partial x_{1}}\right\|_{\mathbf{L}^{6 / 5}\left(\mathbb{R}^{3}\right)}+\left\|\theta_{k_{m}}\right\|_{W_{0}^{1,6 / 5}\left(\mathbb{R}^{3}\right)} \leq C .
\end{aligned}
$$

Therefore $\left(\boldsymbol{w}_{k_{m}}\right)_{m}$ is bounded in $\mathbf{W}_{0}^{2,6 / 5}\left(\mathbb{R}^{3}\right) \cap \mathbf{W}_{0}^{1,12 / 7}\left(\mathbb{R}^{3}\right),\left(\frac{\partial \boldsymbol{w}_{k_{m}}}{\partial x_{1}}\right)_{m}$ is bounded in $\mathbf{L}^{6 / 5}\left(\mathbb{R}^{3}\right),\left(\boldsymbol{w}_{k_{m}}\right)_{m}$ is bounded in $\mathbf{L}^{3}\left(\mathbb{R}^{3}\right)$ and $\left(\theta_{k_{m}}\right)_{m}$ is bounded in $W_{0}^{1,6 / 5}\left(\mathbb{R}^{3}\right)$. Thus, there exist subsequences, again denoted by $\left(\boldsymbol{w}_{k_{m}}\right)_{m}$ and $\left(\theta_{k_{m}}\right)_{m}$, such that $\boldsymbol{w}_{k_{m}} \rightarrow \boldsymbol{w}$ in $\mathbf{W}_{0}^{2,6 / 5}\left(\mathbb{R}^{3}\right) \cap \mathbf{W}_{0}^{1,12 / 7}\left(\mathbb{R}^{3}\right), \frac{\partial \boldsymbol{w}_{k_{m}}}{\partial x_{1}} \rightarrow \frac{\partial \boldsymbol{w}}{\partial x_{1}}$ in $\mathbf{L}^{6 / 5}\left(\mathbb{R}^{3}\right), \boldsymbol{w}_{k_{m}} \rightarrow \boldsymbol{w}$ in $\mathbf{L}^{3}\left(\mathbb{R}^{3}\right)$, and $\theta_{k_{m}} \rightarrow \theta$ in $W_{0}^{1,6 / 5}\left(\mathbb{R}^{3}\right)$. Moreover, since $\mathbf{W}^{2,6 / 5}\left(B_{\rho}\right) \hookrightarrow \mathbf{W}^{1,3 / 2}\left(B_{\rho}\right)$ with compact imbedding, we have $\boldsymbol{w}_{k_{m}} \rightarrow \boldsymbol{w}$ in $\mathbf{W}^{1,3 / 2}\left(B_{\rho}\right)$ with

$$
\|\nabla \boldsymbol{w}\|_{\mathbf{L}^{3 / 2}\left(B_{\rho}\right)}=1,
$$

and

$$
-\nu \Delta \boldsymbol{w}+\lambda \frac{\partial \boldsymbol{w}}{\partial x_{1}}+\boldsymbol{v} \cdot \nabla \boldsymbol{w}+\nabla \theta=0 \text { in } \mathbb{R}^{3} .
$$

Since $\boldsymbol{w} \in \mathbf{W}_{0}^{1,2}\left(\mathbb{R}^{3}\right)$ and $\theta \in L^{2}\left(\mathbb{R}^{3}\right)$, then we have $\Delta \boldsymbol{w}$ and $\nabla \theta$ belonging to $\mathbf{W}_{0}^{-1,2}\left(\mathbb{R}^{3}\right)$. On the other hand, we deduce that $\boldsymbol{v} \cdot \nabla \boldsymbol{w}=\operatorname{div}(\boldsymbol{v} \otimes \boldsymbol{w}) \in$ $\mathbf{W}_{0}^{-1,2}\left(\mathbb{R}^{3}\right)$ because $\boldsymbol{v}$ and $\boldsymbol{w}$ belong to $\mathbf{L}^{4}\left(\mathbb{R}^{3}\right)$. Since $\mathbf{L}^{6 / 5}\left(\mathbb{R}^{3}\right) \hookrightarrow \mathbf{W}_{0}^{-1,2}\left(\mathbb{R}^{3}\right)$ we also have $\frac{\partial \boldsymbol{w}}{\partial x_{1}} \in \mathbf{W}_{0}^{-1,2}\left(\mathbb{R}^{3}\right)$. Hence,

$$
\nu \int_{\mathbb{R}^{3}}|\nabla \boldsymbol{w}|^{2} d \boldsymbol{x}+\left\langle\lambda \frac{\partial \boldsymbol{w}}{\partial x_{1}}+\boldsymbol{v} \cdot \nabla \boldsymbol{w}+\nabla \theta, \boldsymbol{w}\right\rangle_{\mathbf{W}_{0}^{-1,2}\left(\mathbb{R}^{3}\right) \times \mathbf{W}_{0}^{1,2}\left(\mathbb{R}^{3}\right)}=0 .
$$

From (4.5) and (4.22), we deduce $\nabla \boldsymbol{w}=0$ and $\boldsymbol{w}=0$ in $\mathbb{R}^{3}$ which contradicts (4.20). Thanks to (4.15), (4.16) and (4.17), we have the following estimation

$$
\begin{aligned}
& (\lambda \nu)^{1 / 2}\left\|\boldsymbol{w}_{k}\right\|_{\mathbf{L}^{3}\left(\mathbb{R}^{3}\right)}+\lambda^{1 / 4} \nu^{3 / 4}\left\|\nabla \boldsymbol{w}_{k}\right\|_{\mathbf{L}^{12 / 7}\left(\mathbb{R}^{3}\right)} \\
& +\lambda\left\|\frac{\partial \boldsymbol{w}_{k}}{\partial x_{1}}\right\|_{\mathbf{L}^{6 / 5}\left(\mathbb{R}^{3}\right)}+\nu\left\|\nabla^{2} \boldsymbol{w}_{k}\right\|_{\mathbf{L}^{6 / 5}\left(\mathbb{R}^{3}\right)}+\left\|\theta_{k}\right\|_{W_{0}^{1,6 / 5}\left(\mathbb{R}^{3}\right)} \\
& \leq C\left(\|\boldsymbol{f}\|_{\mathbf{L}^{6 / 5\left(\mathbb{R}^{3}\right)}}+\|\boldsymbol{v}\|_{\mathbf{L}^{6}\left(\mathbb{R}^{3}\right)}\|\boldsymbol{f}\|_{\mathbf{L}^{6 / 5}\left(\mathbb{R}^{3}\right)}\right) .
\end{aligned}
$$


We can show that there exist a subsequence of $\left(\boldsymbol{w}_{k}\right)_{k}$ which converges weakly towards $\boldsymbol{w}$ in $\mathbf{W}_{0}^{2,6 / 5}\left(\mathbb{R}^{3}\right) \cap \mathbf{W}_{0}^{1,12 / 7}\left(\mathbb{R}^{3}\right) \cap \mathbf{L}^{3}\left(\mathbb{R}^{3}\right)$ and a subsequence of $\left(\theta_{k}\right)_{k}$ which converges weakly towards $\theta$ in $W_{0}^{1,6 / 5}\left(\mathbb{R}^{3}\right)$ being a solution of the system as follows

$$
-\nu \Delta \boldsymbol{w}+\lambda \frac{\partial \boldsymbol{w}}{\partial x_{1}}+\boldsymbol{v} \cdot \nabla \boldsymbol{w}+\nabla \theta=\boldsymbol{f} \quad \text { and } \quad \operatorname{div} \boldsymbol{w}=0 \text { in } \mathbb{R}^{3} .
$$

We set $\boldsymbol{y}=\boldsymbol{v}-\boldsymbol{w}$ and $\chi=\pi-\theta$. Then we deduce that $(\boldsymbol{y}, \chi)$ is a solution of the following system

$$
-\nu \Delta \boldsymbol{y}+\lambda \frac{\partial \boldsymbol{y}}{\partial x_{1}}+\boldsymbol{v} \cdot \nabla \boldsymbol{y}+\nabla \chi=0 \quad \text { and } \quad \operatorname{div} \boldsymbol{y}=0 \text { in } \mathbb{R}^{3} .
$$

Since $\boldsymbol{y}$ satisfies the energy equality (4.2) with $\boldsymbol{f}=0$, we deduce that $\boldsymbol{y}=\mathbf{0}$ then $\chi=0$. Thanks to uniqueness arguments, we show that $\boldsymbol{w}=\boldsymbol{v}$ and $\theta=\pi$. Theorem is completely proved.

We now search weak solutions of Navier-Stokes system $(\mathcal{N S})$ such that $\boldsymbol{v} \in$ $\mathbf{L}^{q}\left(\mathbb{R}^{3}\right)$ for small values of $q(q<3)$ with similar properties for $\nabla \boldsymbol{v}$. The following theorem allow us to improve the results in Theorem 4.4 by taking an additional assumption for $f$.

Theorem 4.5. Let $1<p<2$. Assume that $\boldsymbol{f} \in \mathbf{L}^{6 / 5}\left(\mathbb{R}^{3}\right) \cap \mathbf{L}^{3 / 2}\left(\mathbb{R}^{3}\right) \cap$ $\mathbf{W}_{0}^{-1, p}\left(\mathbb{R}^{3}\right)$ satisfying the compatibility condition

$$
\forall \boldsymbol{\lambda} \in \mathscr{P}_{\left[1-3 / p^{\prime}\right]}, \quad\left\langle\boldsymbol{f}, \boldsymbol{\lambda}>_{\mathbf{W}_{0}^{-1, p}\left(\mathbb{R}^{3}\right) \times \mathbf{W}_{0}^{1, p^{\prime}}\left(\mathbb{R}^{3}\right)}=0 .\right.
$$

Then each weak solution $(\boldsymbol{u}, \pi)$ to the problem $(\mathcal{N S})$ satisfies (4.12). Besides, we have

$$
\frac{\partial \boldsymbol{v}}{\partial x_{1}} \in \mathbf{W}_{0}^{-1, s}\left(\mathbb{R}^{3}\right) \text { for any } s \geq p \text { and } \pi \in L^{p}\left(\mathbb{R}^{3}\right) .
$$

In particular, if $1<p<\frac{12}{7}$, we obtain additionally

$$
\boldsymbol{v} \in \mathbf{L}^{q}\left(\mathbb{R}^{3}\right) \text { for any } q \geq \frac{4 p}{4-p} \text { and } \nabla \boldsymbol{v} \in \mathbf{L}^{p}\left(\mathbb{R}^{3}\right) .
$$

Proof. Let $\boldsymbol{f} \in \mathbf{W}_{0}^{-1, p}\left(\mathbb{R}^{3}\right)$ with $1<p<2$. From Theorem 4.4 and if $\boldsymbol{u}$ is a solution of $(\mathcal{N S}), \boldsymbol{v}$ satisfies (4.12) and in particular, $\boldsymbol{v} \in \mathbf{L}^{3}\left(\mathbb{R}^{3}\right) \cap \mathbf{L}^{4}\left(\mathbb{R}^{3}\right)$ and $\operatorname{div}(\boldsymbol{v} \otimes \boldsymbol{v}) \in \mathbf{W}_{0}^{-1,3 / 2} \cap \mathbf{W}_{0}^{-1,2}\left(\mathbb{R}^{3}\right)$.

a) The case $3 / 2 \leq p<2$ : We have $\boldsymbol{f}-\boldsymbol{v} \cdot \nabla \boldsymbol{v} \in \mathbf{W}_{0}^{-1, p}\left(\mathbb{R}^{3}\right)$. Thanks to Theorem 1.11 , there exists a unique $(\boldsymbol{w}, \theta)$ such that

$$
-\nu \Delta \boldsymbol{w}+\lambda \frac{\partial \boldsymbol{w}}{\partial x_{1}}+\nabla \theta=\boldsymbol{f}-\boldsymbol{v} \cdot \nabla \boldsymbol{v} \text { and } \operatorname{div} \boldsymbol{w}=0 \text { in } \mathbb{R}^{3},
$$

with $\boldsymbol{w} \in \mathbf{L}^{\frac{4 p}{4-p}}\left(\mathbb{R}^{3}\right) \cap \mathbf{L}^{\frac{3 p}{3-p}}\left(\mathbb{R}^{3}\right), \nabla \boldsymbol{w} \in \mathbf{L}^{p}\left(\mathbb{R}^{3}\right), \frac{\partial \boldsymbol{w}}{\partial x_{1}} \in \mathbf{W}_{0}^{-1, p}\left(\mathbb{R}^{3}\right)$ and $\theta \in$ $L^{p}\left(\mathbb{R}^{3}\right)$. Since $\boldsymbol{v} \in \mathbf{L}^{3}\left(\mathbb{R}^{3}\right)$, by uniqueness arguments, we can deduce that $\boldsymbol{w}=\boldsymbol{v}$, $\theta=\pi$ and then, we have (4.24). 
b) The case $1<p<3 / 2$ : Since $\boldsymbol{f} \in \mathbf{L}^{6 / 5}\left(\mathbb{R}^{3}\right) \hookrightarrow \mathbf{W}_{0}^{-1,2}\left(\mathbb{R}^{3}\right)$, then in particular $\boldsymbol{f} \in \mathbf{W}_{0}^{-1,3 / 2}\left(\mathbb{R}^{3}\right)$ and from the case a), we have $\boldsymbol{v} \in \mathbf{L}^{12 / 5}\left(\mathbb{R}^{3}\right) \cap \mathbf{L}^{3}\left(\mathbb{R}^{3}\right)$. Hence, we can show that $\boldsymbol{v} \cdot \nabla \boldsymbol{v}=\operatorname{div}(\boldsymbol{v} \otimes \boldsymbol{v}) \in \mathbf{W}_{0}^{-1,6 / 5}\left(\mathbb{R}^{3}\right) \cap \mathbf{W}_{0}^{-1,3 / 2}\left(\mathbb{R}^{3}\right)$. We distinguish two following cases:

b1) The case $\frac{6}{5} \leq p<\frac{3}{2}$ : We can prove that $\boldsymbol{f}-\boldsymbol{v} \cdot \nabla \boldsymbol{v} \in \mathbf{W}_{0}^{-1, p}\left(\mathbb{R}^{3}\right)$ satisfying the compatibility condition (4.23). Proceeding as in previous cases, we have

$$
\begin{gathered}
\boldsymbol{v} \in \mathbf{L}^{\frac{4 p}{4-p}}\left(\mathbb{R}^{3}\right) \cap \mathbf{L}^{\frac{3 p}{3-p}}\left(\mathbb{R}^{3}\right), \pi \in L^{p}\left(\mathbb{R}^{3}\right), \\
\nabla \boldsymbol{v} \in \mathbf{L}^{p}\left(\mathbb{R}^{3}\right), \frac{\partial \boldsymbol{v}}{\partial x_{1}} \in \mathbf{W}_{0}^{-1, p}\left(\mathbb{R}^{3}\right) .
\end{gathered}
$$

Hence, we shall gain (4.24) from (4.12). Furthermore, we have (4.25).

b2) The case $p<\frac{6}{5}$ : We have that $f \in \mathbf{W}_{0}^{-1,6 / 5}\left(\mathbb{R}^{3}\right)$ and proceeding as in the case a), we prove that $\boldsymbol{v} \in \mathbf{L}^{q}\left(\mathbb{R}^{3}\right)$ for all $q \geq 12 / 7$. Then, we deduce $\boldsymbol{v} . \nabla \boldsymbol{v}=$ $\operatorname{div}(\boldsymbol{v} \otimes \boldsymbol{v}) \in \mathbf{W}_{0}^{-1, q}\left(\mathbb{R}^{3}\right)$ for all $q>1$ and we obtain $\boldsymbol{f}-\boldsymbol{v} \cdot \nabla \boldsymbol{v} \in \mathbf{W}_{0}^{-1, p}\left(\mathbb{R}^{3}\right)$ satisfying (4.23). Analogously as in the case b1), we can prove that $\boldsymbol{v}$ and $\pi$ satisfy (4.26). Therefore, we have (4.24) and (4.25).

The proof is complete by combining the case a) with the case b).

Thanks to Theorem 3.2 (part ii), Theorem 4.4, Sobolev embedding theorem and the properties of the duality, we can prove the following.

Corollary 4.6. i) Assume that $\boldsymbol{f} \in \mathbf{L}^{p}\left(\mathbb{R}^{3}\right)$ for all $p \in[6 / 5,2)$. Then the Navier-Stokes problem $(\mathcal{N S})$ has a solution $(\boldsymbol{u}, \pi)$ satisfying

$$
\begin{gathered}
\boldsymbol{v} \in \mathbf{L}^{q}\left(\mathbb{R}^{3}\right), \quad \nabla \boldsymbol{v} \in \mathbf{L}^{s_{1}}\left(\mathbb{R}^{3}\right), \pi \in W_{0}^{1, s_{2}}\left(\mathbb{R}^{3}\right), \\
\nabla^{2} \boldsymbol{v} \in \mathbf{L}^{s_{2}}\left(\mathbb{R}^{3}\right), \frac{\partial \boldsymbol{v}}{\partial x_{1}} \in \mathbf{L}^{s_{3}}\left(\mathbb{R}^{3}\right),
\end{gathered}
$$

for any $q \in[3, \infty]$, any $s_{1} \in[12 / 7,6)$, any $s_{2} \in[6 / 5,2)$ and any $s_{3} \in[6 / 5,6)$.

ii) Assume that $\boldsymbol{f} \in \mathbf{L}^{p}\left(\mathbb{R}^{3}\right)$ for all $p \in[6 / 5,3)$. Then we have (4.27) for any $q \in[3, \infty]$, any $s_{1} \in[12 / 7, \infty)$, any $s_{2} \in[6 / 5,3)$ and any $s_{3} \in[6 / 5, \infty)$.

The question can be raise that if we suppose additionally conditions for $\boldsymbol{f}$, then what we shall receive more. We consider the following.

Theorem 4.7. Let $\boldsymbol{f} \in \mathbf{L}^{p}\left(\mathbb{R}^{3}\right)$ for all $p \in(1,3 / 2]$. Then each weak solution $(\boldsymbol{u}, \pi)$ to the problem $(\mathcal{N S})$ satisfies

$$
\begin{gathered}
\boldsymbol{v} \in \mathbf{L}^{q}\left(\mathbb{R}^{3}\right), \quad \nabla \boldsymbol{v} \in \mathbf{L}^{s_{1}}\left(\mathbb{R}^{3}\right), \quad \pi \in W_{0}^{1, s_{2}}\left(\mathbb{R}^{3}\right), \\
\nabla^{2} \boldsymbol{v} \in \mathbf{L}^{s_{2}}\left(\mathbb{R}^{3}\right), \quad \frac{\partial \boldsymbol{v}}{\partial x_{1}} \in \mathbf{L}^{s_{3}}\left(\mathbb{R}^{3}\right),
\end{gathered}
$$

for any $q \in(2, \infty)$, any $s_{1} \in(4 / 3,3]$, any $s_{2} \in(1,3 / 2]$ and any $s_{3} \in(1,3]$.

Proof. Remark that if $\boldsymbol{f} \in \mathbf{L}^{6 / 5}\left(\mathbb{R}^{3}\right) \cap \mathbf{L}^{3 / 2}\left(\mathbb{R}^{3}\right)$, from Theorem 4.4, we can deduce that $\boldsymbol{f}-\boldsymbol{v} . \nabla \boldsymbol{v} \in \mathbf{L}^{12 / 11}\left(\mathbb{R}^{3}\right)$. From Theorem 1.10 with $p=\frac{12}{11}$ and proceeding as in the proof of Theorem 3.1, we obtain $\boldsymbol{v} \in \mathbf{L}^{12 / 5}\left(\mathbb{R}^{3}\right) \cap \mathbf{L}^{12 / 7}\left(\mathbb{R}^{3}\right), \nabla \boldsymbol{v} \in$ 
$\mathbf{L}^{4 / 3}\left(\mathbb{R}^{3}\right) \cap \mathbf{L}^{12 / 7}\left(\mathbb{R}^{3}\right), \nabla^{2} \boldsymbol{v}$ and $\frac{\partial \boldsymbol{v}}{\partial x_{1}}$ belong to $\mathbf{L}^{12 / 11}\left(\mathbb{R}^{3}\right), \pi \in W_{0}^{1,12 / 11}\left(\mathbb{R}^{3}\right)$. Combining with the results in Theorem 4.4, we have $\boldsymbol{v} \in \mathbf{L}^{q}\left(\mathbb{R}^{3}\right)$ for all $q \in$ $[12 / 5, \infty)$ and $\nabla \boldsymbol{v} \in \mathbf{L}^{4 / 3}\left(\mathbb{R}^{3}\right) \cap \mathbf{L}^{3}\left(\mathbb{R}^{3}\right)$. Hence, it is easy to prove that $\boldsymbol{f}-\boldsymbol{v} \cdot \nabla \boldsymbol{v}$ belongs to $\mathbf{L}^{p}\left(\mathbb{R}^{3}\right)$. Thanks to Theorem 1.10 for all $p \in(1,3 / 2]$, we can deduce that $\boldsymbol{v} \in \mathbf{L}^{\frac{2 p}{2-p}}\left(\mathbb{R}^{3}\right) \cap \mathbf{L}^{\frac{3 p}{3-p}}\left(\mathbb{R}^{3}\right), \nabla \boldsymbol{v} \in \mathbf{L}^{\frac{4 p}{4-p}}\left(\mathbb{R}^{3}\right) \cap \mathbf{L}^{\frac{3 p}{3-p}}\left(\mathbb{R}^{3}\right), \nabla^{2} \boldsymbol{v} \in \mathbf{L}^{p}\left(\mathbb{R}^{3}\right)$, $\frac{\partial \boldsymbol{v}}{\partial x_{1}} \in \mathbf{L}^{p}\left(\mathbb{R}^{3}\right)$ and $\pi \in W_{0}^{1, p}\left(\mathbb{R}^{3}\right)$. Clearly, we have (4.28) by combining with (4.12).

Thanks to Corollary 4.6 and Theorem 4.7, we obtain the following results.

Corollary 4.8. i) Assume that $\boldsymbol{f} \in \mathbf{L}^{p}\left(\mathbb{R}^{3}\right)$ for all $1<p<2$. Then each weak solution $(\boldsymbol{u}, \pi)$ to $(\mathcal{N S})$ satisfies

$$
\begin{gathered}
\boldsymbol{v} \in \mathbf{L}^{q}\left(\mathbb{R}^{3}\right), \quad \nabla \boldsymbol{v} \in \mathbf{L}^{s_{1}}\left(\mathbb{R}^{3}\right), \pi \in W_{0}^{1, s_{2}}\left(\mathbb{R}^{3}\right), \\
\nabla^{2} \boldsymbol{v} \in \mathbf{L}^{s_{2}}\left(\mathbb{R}^{3}\right), \frac{\partial \boldsymbol{v}}{\partial x_{1}} \in \mathbf{L}^{s_{3}}\left(\mathbb{R}^{3}\right),
\end{gathered}
$$

for any $q \in(2, \infty]$, any $s_{1} \in[4 / 3,6)$, any $s_{2} \in[1,2)$ and any $s_{3} \in[1,6)$.

ii) Assume that $\boldsymbol{f} \in \mathbf{L}^{p}\left(\mathbb{R}^{3}\right)$ for all $1<p<3$. Then we have (4.29) for any $q \in(2, \infty]$, any $s_{1} \in(4 / 3, \infty)$, any $s_{2} \in(1,3)$ and any $s_{3} \in(1, \infty)$.

In Theorem 4.7, we know that if $\boldsymbol{f} \in \mathbf{L}^{p}\left(\mathbb{R}^{3}\right)$ for all $p \in(1,3 / 2]$, then $\boldsymbol{v}$ satisfies (4.28). With additional assumption for $f$, we shall prove that the weak solutions given in Theorem 4.7 satisfy better properties.

Theorem 4.9. Given $r>1$. Assume that $\boldsymbol{f} \in \mathbf{L}^{p}\left(\mathbb{R}^{3}\right) \cap \mathbf{W}_{0}^{-1, r}\left(\mathbb{R}^{3}\right)$ for all $p \in(1,3 / 2]$ satisfying the compatibility condition

$$
\forall \boldsymbol{\lambda} \in \mathscr{P}_{\left[1-3 / r^{\prime}\right]}, \quad\left\langle\boldsymbol{f}, \boldsymbol{\lambda}>_{\mathbf{W}_{0}^{-1, r}\left(\mathbb{R}^{3}\right) \times \mathbf{W}_{0}^{1, r^{\prime}}\left(\mathbb{R}^{3}\right)}=0 .\right.
$$

Then each weak solution $(\boldsymbol{u}, \pi)$ to $(\mathcal{N S})$ satisfies (4.28) and $\frac{\partial \boldsymbol{v}}{\partial x_{1}} \in \mathbf{W}_{0}^{-1, s}\left(\mathbb{R}^{3}\right)$ for any $s \geq r$. Moreover,

$$
\begin{gathered}
\text { if } 1<r \leq \frac{3}{2}, \pi \in L^{t}\left(\mathbb{R}^{3}\right) \text { for all } r \leq t \leq 3, \\
\text { if } 1<r \leq \frac{4}{3}, \boldsymbol{v} \in \mathbf{L}^{q}\left(\mathbb{R}^{3}\right) \text { for all } q \geq \frac{4 r}{4-r} \text { and } \nabla \boldsymbol{v} \in \mathbf{L}^{r}\left(\mathbb{R}^{3}\right) \text {. }
\end{gathered}
$$

Proof. We know that $(\boldsymbol{u}, \pi)$ satisfies (4.28). In addition, thanks to Theorem 4.7, we have $\boldsymbol{v} \otimes \boldsymbol{v} \in \mathbf{L}^{q}\left(\mathbb{R}^{3}\right)$ for all $q>1$ and

$$
\boldsymbol{f}-\operatorname{div}(\boldsymbol{v} \otimes \boldsymbol{v}) \in \mathbf{W}_{0}^{-1, r}\left(\mathbb{R}^{3}\right) \perp \mathscr{P}_{\left[1-3 / r^{\prime}\right]} .
$$

Hence, thanks to Theorem 1.10, it is easy to prove that $\boldsymbol{v} \in \mathbf{L}^{\frac{4 r}{4-r}}\left(\mathbb{R}^{3}\right) \cap \mathbf{L}^{\frac{3 r}{3-r}}\left(\mathbb{R}^{3}\right)$, $\nabla \boldsymbol{v} \in \mathbf{L}^{r}\left(\mathbb{R}^{3}\right), \frac{\partial \boldsymbol{v}}{\partial x_{1}} \in \mathbf{W}_{0}^{-1, r}\left(\mathbb{R}^{3}\right)$ and $\pi \in L^{r}\left(\mathbb{R}^{3}\right)$. As $\boldsymbol{v} \in \mathbf{L}^{q}\left(\mathbb{R}^{3}\right)$ for any $q \geq 2$, we have $\frac{\partial \boldsymbol{v}}{\partial x_{1}} \in \mathbf{W}_{0}^{-1, s}\left(\mathbb{R}^{3}\right)$ for any $s \geq r$. Remark that $\frac{4 r}{4-r} \leq 2$ if $r \leq \frac{4}{3}$, then we obtain (4.31). For the pressure, we note that thanks to (4.28), $\pi \in L^{t}\left(\mathbb{R}^{3}\right)$ for all $3 / 2<t \leq 3$ and then, we have (4.30). The Theorem is completely proved. 
We now prove the following theorem.

Theorem 4.10. Let $1<p<\infty$ and $q_{0} \geq 3$. Assume that $\boldsymbol{f} \in \mathbf{L}^{q}\left(\mathbb{R}^{3}\right) \cap$ $\mathbf{W}_{0}^{-1, p}\left(\mathbb{R}^{3}\right)$ for all $q \in\left(1, q_{0}\right]$ and satisfying the compatibility condition

$$
\forall \boldsymbol{\lambda} \in \mathscr{P}_{\left[1-3 / p^{\prime}\right]}, \quad\langle\boldsymbol{f}, \boldsymbol{\lambda}\rangle_{\mathbf{W}_{0}^{-1, p}\left(\mathbb{R}^{3}\right) \times \mathbf{W}_{0}^{1, p^{\prime}}\left(\mathbb{R}^{3}\right)}=0 .
$$

Then the problem $(\mathcal{N S})$ has a solution $(\boldsymbol{u}, \pi)$ satisfying

$$
\begin{gathered}
\boldsymbol{v} \in \mathbf{L}^{s_{0}}\left(\mathbb{R}^{3}\right), \quad \nabla \boldsymbol{v} \in \mathbf{L}^{s_{1}}\left(\mathbb{R}^{3}\right), \pi \in W_{0}^{1, s_{2}}\left(\mathbb{R}^{3}\right), \\
\nabla^{2} \boldsymbol{v} \in \mathbf{L}^{s_{2}}\left(\mathbb{R}^{3}\right), \frac{\partial \boldsymbol{v}}{\partial x_{1}} \in \mathbf{L}^{s_{3}}\left(\mathbb{R}^{3}\right),
\end{gathered}
$$

for all $s_{0} \in(2, \infty], s_{1} \in(4 / 3, \infty), s_{2} \in\left(1, q_{0}\right], s_{3} \in(1, \infty)$. In particular, if $1<p \leq 3 / 2$, we have additionally $\pi \in L^{k_{1}}\left(\mathbb{R}^{3}\right)$ for any $k_{1} \geq p$. Moreover, if $1<p \leq 4 / 3$, we obtain $\boldsymbol{v} \in \mathbf{L}^{k_{2}}\left(\mathbb{R}^{3}\right)$ for any $k_{2} \in\left[\frac{4 p}{4-p}, \infty\right]$ and $\nabla \boldsymbol{v} \in \mathbf{L}^{k_{3}}\left(\mathbb{R}^{3}\right)$ for any $k_{3} \geq p$.

Proof. In particular, we have $\boldsymbol{f} \in \mathbf{L}^{q}\left(\mathbb{R}^{3}\right)$ for all $1<q<3$. From Corollary 4.8 part ii), we have

$$
\begin{gathered}
\boldsymbol{v} \in \mathbf{L}^{s_{0}}\left(\mathbb{R}^{3}\right), \quad \nabla \boldsymbol{v} \in \mathbf{L}^{s_{1}}\left(\mathbb{R}^{3}\right), \pi \in W_{0}^{1, s_{2}}\left(\mathbb{R}^{3}\right), \\
\nabla^{2} \boldsymbol{v} \in \mathbf{L}^{s_{2}}\left(\mathbb{R}^{3}\right), \frac{\partial \boldsymbol{v}}{\partial x_{1}} \in \mathbf{L}^{s_{3}}\left(\mathbb{R}^{3}\right),
\end{gathered}
$$

for any $s_{0} \in(2, \infty]$, any $s_{1} \in(4 / 3, \infty)$, any $s_{2} \in(1,3)$ and any $s_{3} \in(1, \infty)$. Then, we deduce that $\boldsymbol{f}-\boldsymbol{v} . \nabla \boldsymbol{v} \in \mathbf{L}^{q}\left(\mathbb{R}^{3}\right)$ for all $q \in\left(1, q_{0}\right]$ and we can obtain that $\pi \in W_{0}^{1, q}\left(\mathbb{R}^{3}\right), \nabla^{2} \boldsymbol{v} \in \mathbf{L}^{q}\left(\mathbb{R}^{3}\right), \frac{\partial \boldsymbol{v}}{\partial x_{1}} \in \mathbf{L}^{q}\left(\mathbb{R}^{3}\right)$. Combining with the previous results, we have (4.32) for all $s_{2} \in\left(1, q_{0}\right], s_{3} \in(1, \infty)$. As $\boldsymbol{v} \otimes \boldsymbol{v} \in \mathbf{L}^{r}\left(\mathbb{R}^{3}\right)$ for any $r>1$, then

$$
\boldsymbol{f}-\boldsymbol{v} . \nabla \boldsymbol{v} \in \mathbf{W}_{0}^{-1, p}\left(\mathbb{R}^{3}\right) \perp \mathscr{P}_{\left[1-3 / p^{\prime}\right]} .
$$

If $1<p<3$, from Theorem 1.11, the Oseen system (3.4) has a unique solution $(\boldsymbol{w}, \theta) \in\left(\widetilde{\mathbf{W}}_{0}^{1, p}\left(\mathbb{R}^{3}\right) \times L^{p}\left(\mathbb{R}^{3}\right)\right)$ such that $\boldsymbol{w} \in \mathbf{L}^{s}\left(\mathbb{R}^{3}\right)$ for all $\frac{4 p}{4-p} \leq s \leq \frac{3 p}{3-p}$. We use the same technique in the proof of Theorem 3.1, we deduce that $\boldsymbol{w}=\boldsymbol{v}$ and $\theta=\pi$. Note that $\pi \in L^{k_{1}}\left(\mathbb{R}^{3}\right)$ for any $k_{1} \geq p$ if $1<p \leq 3 / 2$. Moreover, if $1<p \leq 4 / 3$, we can deduce $\frac{4 p}{4-p} \leq 2$, then $\boldsymbol{v} \in \mathbf{L}^{k_{2}}\left(\mathbb{R}^{3}\right)$ for any $k_{2} \in\left[\frac{4 p}{4-p}, \infty\right]$ and $\nabla \boldsymbol{v} \in \mathbf{L}^{k_{3}}\left(\mathbb{R}^{3}\right)$ for any $k_{3} \geq p$. The Theorem is completely proved.

We now consider the energy identity. The key idea to find the conditions to obtain the energy identity (4.33), is to test the Navier-Stokes problem with $\boldsymbol{v}$. Following this idea, we can deduce the following theorem.

Theorem 4.11. Let $\boldsymbol{f} \in \mathbf{L}^{6 / 5}\left(\mathbb{R}^{3}\right) \cap \mathbf{L}^{3 / 2}\left(\mathbb{R}^{3}\right)$ and $(\boldsymbol{u}, \pi)$ be a weak solution of $(\mathcal{N S})$. Then we have the energy identity

$$
\nu \int_{\mathbb{R}^{3}}|\nabla \boldsymbol{v}|^{2} d \boldsymbol{x}=\langle\boldsymbol{f}, \boldsymbol{v}\rangle_{\mathbf{W}_{0}^{-1,2}\left(\mathbb{R}^{3}\right) \times \mathbf{W}_{0}^{1,2}\left(\mathbb{R}^{3}\right)} .
$$


Proof. Thanks to Theorem 4.4, we have that $\boldsymbol{v} \in \mathbf{L}^{4}\left(\mathbb{R}^{3}\right) \cap \mathbf{W}_{0}^{1,2}\left(\mathbb{R}^{3}\right), \frac{\partial \boldsymbol{v}}{\partial x_{1}} \in$ $\mathbf{W}_{0}^{-1,2}\left(\mathbb{R}^{3}\right)$ and $\pi \in L^{2}\left(\mathbb{R}^{3}\right)$. As in Lemma 4.1 , we show that

$$
\left\langle\lambda \frac{\partial \boldsymbol{v}}{\partial x_{1}}+\boldsymbol{v} \cdot \nabla \boldsymbol{v}+\nabla \pi, \boldsymbol{v}\right\rangle_{\mathbf{W}_{0}^{-1,2}\left(\mathbb{R}^{3}\right) \times \mathbf{W}_{0}^{1,2}\left(\mathbb{R}^{3}\right)}=0 .
$$

and we obtain the energy identity (4.33).

\section{References}

[1] R. A. Adams, Sobolev Spaces, Academic Press, New York, (2003).

[2] F. Alliot, C. Amrouche, On the regularity and decay of the weak solutions to the steady-state Navier-Stokes equations in exterior domains, Appl. Nonlinear Analysis, Edited by A. Sequeira, H. Beirao da Veiga and J. H. Videman, Kluwer Academic, 1-18 (1999).

[3] F. Alliot, C. Amrouche, The Stokes problem in $\mathbb{R}^{3}$ : an approach in weighted Sobolev spaces, Math. Meth. Mod. Appl. Sci. , 9, 723-754, (1999).

[4] C. Amrouche, V. Girault, Decomposition of vector spaces and application to the Stokes problem in arbitrary dimension, Czechoslovak Mathematical Journal, Praha, 44 (119), 109-140, (1994).

[5] C. Amrouche, V. Girault, J. Giroire, Weighted Sobolev spaces for the Laplace equation in $\mathbb{R}^{n}$, J. Math. Pures et Appl., 73 (6), 579-606, (1994).

[6] C. Amrouche, V. Girault, J. Giroire, Dirichlet and Neumann exterior problems for the n-dimensional Laplace operator: an approach in weighted Sobolev spaces, J. Math. Pures et Appl., 76 (1), 55-81, (1997).

[7] C. Amrouche, U. Razafison, The stationary Oseen equations in $\mathbb{R}^{3}$. An approach in weighted Sobolev spaces, J. Math. Fluids Mech., (online: DOI $10.1007 / \mathrm{s} 00021-005-0197-\mathrm{z})$.

[8] C. Amrouche, U. Razafison, Weighted Sobolev spaces for a scalar model of the stationary Oseen equations in $\mathbb{R}^{n}$, J. Math. Fluids Mech., (online: DOI 10.1007/s00021-005-0195-1).

[9] K. I. BABEnKo, On stationary solutions of the problem of flow past a body of a viscous incompressible fluid, Math. USSR, Sb. , 20, 1-25, (1973).

[10] R. FARWIG, The stationary Navier-Stokes equations in a 3D-exterior domain, Lecture Notes in Numer. Appl. Anal., 16, 53-115, (1998).

[11] G. P. GALDI, An introduction to the mathematical theory of the NavierStokes equations, vol I and vol II, Springer tracts in natural philosophy, (1994).

[12] V. Girault, P-A. Raviart, Finite element methods for Navier-Stokes equations (Theory and Algorithms), Springer-Verlag Berlin Heidelberg, (1986). 
[13] J. LeRAy, Sur le mouvement d'un liquide visqueux emplissant l'espace, Acta Math., 63, 193-248, (1934). 